\title{
29. GEOCHEMISTRY OF ORGANIC CARBON IN SOUTH ATLANTIC SEDIMENTS FROM DEEP SEA DRILLING PROJECT LEG 751
}

\author{
Philip A. Meyers, Oceanography Program, Department of Atmospheric and Oceanic Science, The University of \\ Michigan, Ann Arbor, Michigan \\ Simon C. Brassell, Organic Geochemistry Unit, School of Chemistry, University of Bristol, \\ Bristol, BS8 1TS, England \\ and \\ Alain Y. Huc, ${ }^{2}$ Laboratoire de Géologique Appliquée, Université D’Orlèans, 45046 Orléans, France
}

\begin{abstract}
CHN analyses of sediments and rocks sampled during DSDP Leg 75 in the South Atlantic have provided concentrations of organic carbon and atomic $\mathrm{C} / \mathrm{N}$ ratios of organic matter from two sites. High values of organic carbon were measured in sediments deposited during Neogene and Cretaceous times at Site 530 in the Angola Basin; sediments deposited at other times contain less than $0.5 \%$ organic carbon. Development of the Benguela Current and associated upwelling-supported biological productivity is recorded in late Miocene to Holocene sediments which contain 1 to $7 \%$ organic carbon. These sediments include debris flows and turbidites composed of predominantly biogenic materials originally deposited on the Walvis Ridge and on the African continental margin. Organic-carbon-rich black shales containing up to $17 \%$ organic carbon occur in late Albian to Coniacian turbidite sequences. These Cretaceous black shale layers are commonly several centimeters thick and are separated by bioturbated fine-grained organic-carbon-poor turbidites which are usually much thicker. At Site 532 on the Walvis Ridge, biogenic sediments deposited between late Miocene and Holocene times contain 1 to $9 \%$ organic carbon. Fluctuations in the intensity of high biological productivity associated with the Benguela Current are preserved in alternating light and dark layers of sediments. $\mathrm{C} / \mathrm{N}$ ratios of organic matter in sediments from both sites are typical of marine sources.
\end{abstract}

\section{INTRODUCTION}

The composition of organic matter in marine sediments is determined largely by the biological productivity of overlying waters, the oxygen content of bottom waters, and the extent of diagenesis in sediments. A fundamental parameter of organic geochemical investigations of sediments is the organic carbon content, which is directly related to the amount of organic matter present. This quantity reflects the depositional environment of the sediment because the degree of preservation of organic matter is determined by interrelated physical, chemical, and biological factors. Availability of marineand land-derived organic matter, oxicity/anoxicity of the depositional environment, and the sedimentation rate are major factors affecting the quantity of organic carbon that is incorporated into sediments. These factors do not, however, completely determine the ultimate organic carbon content of a sediment because diagenetic modification and degradation of organic matter takes place after burial.

Although the factors governing the organic nitrogen content of sediments are less well understood than those controlling the amount of organic carbon present, generally similar criteria apply. A useful relative measure of the nitrogen content of sediment organic matter is the $\mathrm{C} / \mathrm{N}$ atomic ratio. Values between 14 and 30 are charac-

\footnotetext{
${ }^{1}$ Hay, W. W., Sibuet, J.-C., et al., Init. Repts. DSSDP, 75: Washington (U.S. Govt. Printing Office).

${ }^{2}$ Present address: Départment de Physico-Chimie, Institut Français du Pétrole, 92506 Rueil-Malmaison, France.
}

teristic of terrigenous material, whereas those between 4 and 8 typify marine organic matter (Goodell, 1972; Müller, 1977). Changes during diagenesis significantly affect the precise value of the $\mathrm{C} / \mathrm{N}$ ratio and therefore may limit its utility (Müller, 1977; Waples and Sloan, 1980).

During Deep Sea Drilling Project (DSDP), Leg 75, measurements of organic carbon content and of organic $\mathrm{C} / \mathrm{N}$ ratios were done on sediment samples from two locations in the southeastern Atlantic Ocean (Fig. 1). Site 530 is in $4629 \mathrm{~m}$ water depth at the eastern margin of the Angola Basin, not far from the northern flank of the Walvis Ridge. Site 532 is on the Walvis Ridge at a water depth of $1331 \mathrm{~m}$ and close to Site 362 of Leg 40.

At Site 530, sediments were obtained from $115 \mathrm{~m}$ sub-bottom to basement at $1103 \mathrm{~m}$ by rotary drilling in Hole $530 \mathrm{~A}$ and from the water/sediment interface to $181 \mathrm{~m}$ sub-bottom by hydraulic piston coring (HPC) in Hole 530B. Two HPC holes, 532 and 532B, provided sediments to a total sub-bottom depth of $291 \mathrm{~m}$ at Site 532. Samples for organic analysis were taken routinely throughout these sediment sequences. We report here the results of organic carbon and organic $\mathrm{C} / \mathrm{N}$ ratio determinations done onboard D/V Glomar Challenger and augmented by later analyses done at the University of Michigan.

\section{ANALYSIS}

\section{Determination of Calcium Carbonate Content}

The $\mathrm{CaCO}_{3}$ contents of sediment samples were determined onboard ship by the "karbonate bomb" technique (Müller and Gastner, 1971). In this procedure, a dried sample is powdered, and a weighed 


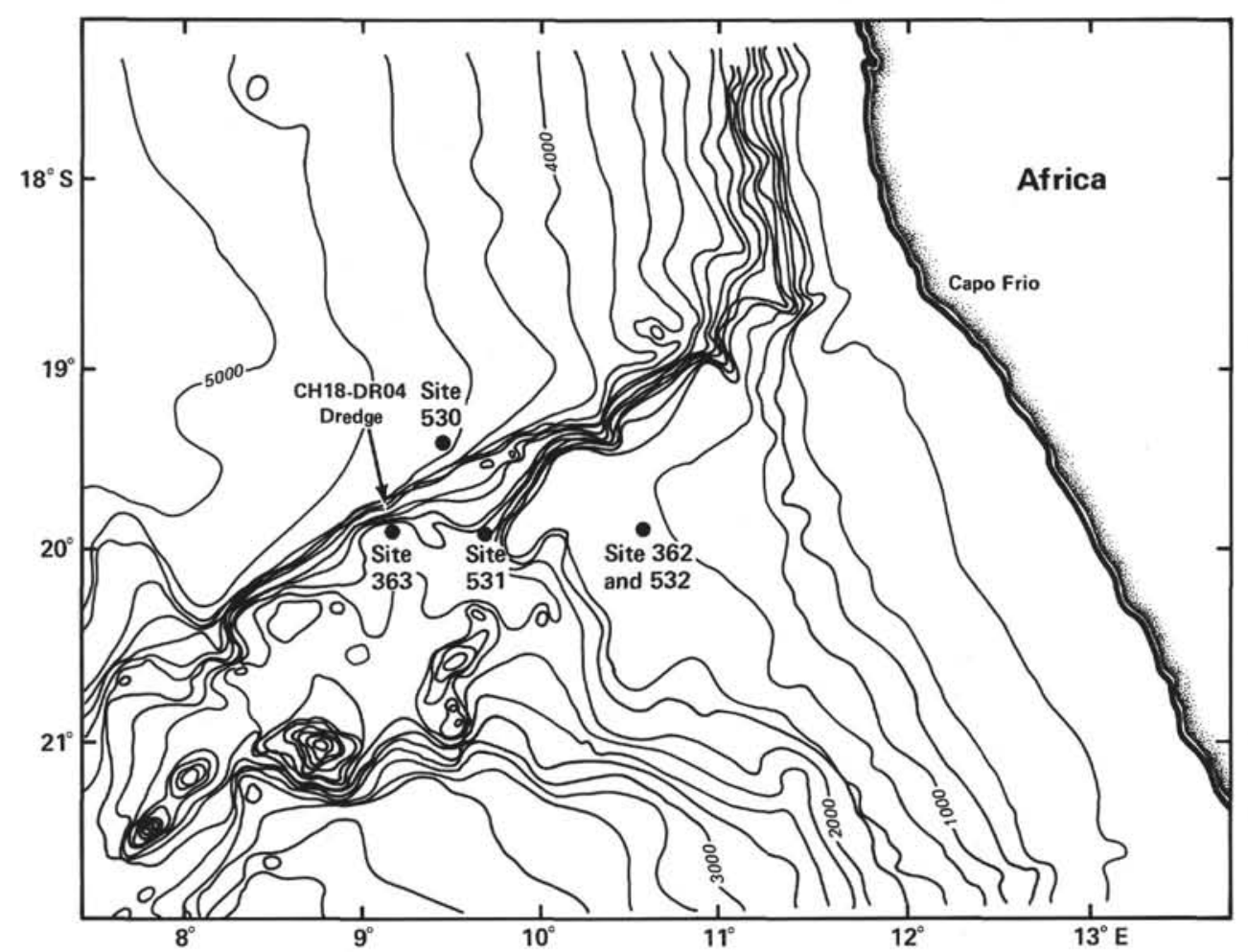

Figure 1. Locations of Site 530 (rotary drilling and hydraulic piston coring) and Site 532 (hydraulic piston coring). (Depth contours in $\mathrm{m}$.)

aliquot is reacted with $\mathrm{HCl}$ in a closed pressure bomb. Any resulting $\mathrm{CO}_{2}$ pressure is proportional to the $\mathrm{CaCO}_{3}$ content of the sample. Application of a calibration factor to the manometer reading yields percent $\mathrm{CaCO}_{3}$. Percent error can be as low as $1 \%$ for sediments high in $\mathrm{CaCO}_{3}$, and in general an accuracy of \pm 2 to $5 \%$ can be obtained.

Shorebased $\mathrm{CaCO}_{3}$ determinations were done by measuring the total carbon content of sediments before and after acid treatment with $\mathrm{HCl}$. A Hewlett-Packard 185B CHN analyzer was used for the carbon measurements, and the percent $\mathrm{CaCO}_{3}$ was calculated from the difference between initial and residual carbon contents.

\section{Determination of Organic Carbon and Nitrogen Content}

Shipboard organic carbon analyses were done using a HewlettPackard 185B CHN analyzer with He carrier gas at 60 psi $(120$ $\mathrm{ml} / \mathrm{min}$.). Portions of dried and ground samples selected from each core for $\mathrm{CaCO}_{3}$ analysis were treated with dilute $\mathrm{HCl}$ to remove carbonate, washed with deionized water, and dried at $110^{\circ} \mathrm{C}$. A Cahn electrobalance, mounted on a gimbal, was used to weigh $20 \mathrm{mg}$ samples of sediment for $\mathrm{CHN}$ analysis. The balance has an estimated accuracy of $\pm 0.2 \mathrm{mg}$, depending upon sea conditions. Samples were combusted at $1050^{\circ} \mathrm{C}$ with preconditioned oxidant $(25 \mathrm{mg})$, and the volumes of the evolved gases determined by gas chromatography as measures of the $\mathrm{C}, \mathrm{H}$, and $\mathrm{N}$ contents of sedimentary organic matter. Areas of gas peaks were determined with a Columbia Scientific Industries (CSI) mini-lab integrator and compared to those of standards of known organic carbon content (Table 1). Concentrations of $\mathrm{H}$ and $\mathrm{N}$ are not known in most of these standards. For USGS SDO-1, Leventhal et al. (1978) report total $\mathrm{N}$ of $0.34 \%$ and organic $\mathrm{N}$ of $0.21 \%$. These values were used to standardize instrument response so that $\mathrm{C} / \mathrm{N}$ atomic ratios could be reported. This approach differs from earlier shipboard procedures which used a synthetic $\mathrm{CHN}$ standard to determine nitrogen response factors. Values for hydrogen contents obtained by this CHN method are untrustworthy because of variable amounts of clay minerals and their hydrates and are not reported here.

Organic carbon analyses were repeated on shore for a number of the samples analyzed onboard D/V Glomar Challenger. In addition, many of the shipboard $\mathrm{CaCO}_{3}$ samples were analyzed for organic car-
Table 1. Standards used during Leg 75 for organic carbon determinations.

\begin{tabular}{lccl}
\hline Standard & $\begin{array}{c}\text { Total C } \\
(\%)\end{array}$ & $\begin{array}{c}\text { Organic C } \\
(\%)\end{array}$ & \multicolumn{1}{c}{ Source } \\
\hline AWM & 0.45 & 0.27 & Phillips Petroleum Company \\
AVB & 0.57 & 0.45 & Phillips Petroleum Company \\
27251 & 2.68 & 2.48 & Institut Français du Pétrole \\
$66-488-16$ & 2.97 & 2.50 & DSDP \\
$63-467-58-3$ & 8.90 & 4.31 & DSDP \\
SDO-1 & 10.40 & 10.06 & U.S. Geological Survey \\
\hline
\end{tabular}

bon and nitrogen to provide new data. Sample preparation and instrumentation were essentially the same as those used onboard ship.

Organic carbon determinations done on the same samples onboard D/V Glomar Challenger and at The University of Michigan agree well. The mean difference between the shipboard and shorebased organic carbon percentages is \pm 0.32 absolute percent. The overall relative deviation from the mean of each shipboard-shorebased comparison is $\pm 8.87 \%$, but this indicator of analytical precision improves to $\pm 4.37 \%$ for organic carbon concentrations above $1 \%$ dry sediment weight. Problems with determination of organic nitrogen at The University of Michigan appear as poor agreement in atomic $\mathrm{C} / \mathrm{N}$ ratios. The greater consistency of $\mathrm{C} / \mathrm{N}$ ratios calculated from shipboard analyses suggests that these values are more reliable then the shorebased nitrogen analyses, hence discussion of $\mathrm{C} / \mathrm{N}$ ratios will be based upon the shipboard data.

\section{LITHOLOGIC SETTINGS}

\section{Site 530-Angola Basin}

A nearly continuous record of sedimentation in the Angola basin was obtained by drilling in Hole 530A and 
hydraulic piston coring in Hole 530B. The sequences of lithologies encountered in Holes 530A and 530B are shown in Figure 2 and are fully described in the Site 530 summary (this volume).

Unit 1 consists of Pleistocene sediments with varying proportions of nannofossils, diatoms, and clay. Colors of all sediments are shades of olive and olive gray; darker sediments are generally more diatom-rich and lighter sediments are generally more nannofossil-rich. Unit 1 is subdivided into two subunits on the basis of relative abundances of diatoms and nannofossils. The distinction between Subunits $1 \mathrm{a}$ and $1 \mathrm{~b}$ is the decrease in abundance of nannofossils to nil and the increase in relative abundance of diatoms to more than $40 \%$ in most of Subunit 1b. Debris-flow deposits and turbidites are abundant throughout Unit 1.

Unit 2, Pleistocene to late Miocene in age, consists mainly of calcareous biogenic sediments interbedded with thick debris-flow deposits and thin mud turbidites. Colors of the sediments range from light greenish gray to olive to olive gray; the darker colors reflect an increasing clay content. The biogenic sediments are composed dominantly of nannofossils, with variable contents of foraminifers and clay and rare siliceous material.

Unit 3 consists of very soft, interbedded red and green middle Miocene to Oligocene muds, with rare, thin layers of dark greenish black turbidite silts and muds, some scattered layers of volcanic ash, and rare foraminifer-nannofossil ooze. There is little compositional difference between green and red muds. Both contain a high proportion of clay-sized material, and the green coloration appears often to be the result of reduction of iron in the red mud (Sibuet, Hay et al., this volume).

Unit 4, Eocene to Maestrichtian in age, is made up of mudstone, calcareous mudstone, and marlstone, commonly with interbeds of nannofossil chalk and clastic limestone. Green-colored components are dominant, but colors grade into red. The colors vary with the proportions of carbonate present-white and bluish white limestone; yellowish gray chalk; greenish and olive gray mudstone; dark greenish gray mudstone. The rock layers in Unit 4 tend to occur as turbidites with a sharp bases and bioturbated tops.

The dominant lithologies of Unit 5 are Maestrichtian to Campanian green mudstone and marlstone, reflecting varying proportions of silt, clay, and carbonate. Interbeds of clastic limestone are present in the upper part of the unit but become less common downward as carbonate clastic debris is replaced by dark-colored siliciclastics. Unit 5 is divided into three subunits on the basis of abundance and composition of coarse clastics. In Subunit 5A, well-cemented clastic turbidites are commonly interbedded with greenish-gray mudstone. The concentration of carbonate is somewhat lower in Subunit $5 \mathrm{~b}$ than in Subunit $5 \mathrm{a}$ because of the introduction of sandstone beds and a decrease in number and thickness of clastic limestone beds downward within the subunit. In Subunit 5c, sand replaces clastic carbonate in medium-bedded, well-defined turbidites of calcareous sandstone. Bioturbation is common throughout Unit 5 .

The dominant lithology of Unit 6 is carbonate-cemented, greenish black volcanogenic sandstone deposited as graded turbidites. The dark green mudstone tops of these turbidites are commonly overlain by beds of lighter green, bioturbated, calcareous mudstone or marlstone. This unit is Campanian in age.

Unit 7 is Campanian to Santonian in age and consists of red claystone with interbeds of green, red, and purple siltstone and claystone and green sandstone in numerous repeated turbidite sequences. As sandstone becomes less abundant, the thickness of individual turbidites decreases downward to the base of this unit, where most of the turbidites consist only of reddish purple and red siltstone and claystone.

Unit 8 is composed of Coniacian to Albian red and green claystones like those at the base of Unit 7 with the addition of thin layers of black shale. The claystone beds occasionally contain thin green siltstone turbidite layers and are usually bioturbated, although many of the red claystone beds appear to be massive. Pyrite is commonly associated with both green claystone and black shale as disseminated crystals and as large aggregates.

Unit 9 consists of medium gray, fine-grained Albian basalt. The contact between the basalt and red mudstone of Unit 8 occurs in the core catcher of Core 530A105.

From Early Cretaceous (Albian) to Eocene time, the dominant sediments supplied to the southern Angola Basin were fine-grained distal turbidities derived from the African continental margin. Beginning in the Late Cretaceous (Coniacian), a small amount of coarser turbidites of varying composition were also supplied. These sediments contain a variable, mostly minor carbonate component from both pelagic and continental margin sources, which indicates that the site was generally above the calcite compensation depth (CCD).

Mud turbidites deposited from the late Oligocene through the late Miocene indicate that turbidity currents were still supplying fine-grained material from the African continental margin although the supply of coarseclastic debris had stopped. The almost complete lack of carbonate suggests that during this time (30 to $10 \mathrm{~m}$.y. ago) the site was below the CCD.

A marked increase in carbonate accumulation, seen as nannofossil marl and ooze, began in the late Miocene. This probably records the rapid deepening of the CCD resulting from increased productivity. Turbidity currents still supplied fine-grained clastics that formed part of the clay-marl-ooze cycles, but most of the sediment that accumulated was nannofossil debris. The first indication of sediment supplied from Walvis Ridge is recorded by the debris-flow deposits that began to accumulate during the late Miocene. Increased diatom productivity during the Pliocene resulted in the accumulation of diatom-rich, carbonate-poor sediments interbedded with ridge-derived debris-flow deposits. An increase in nannofossil abundance followed by an increase 

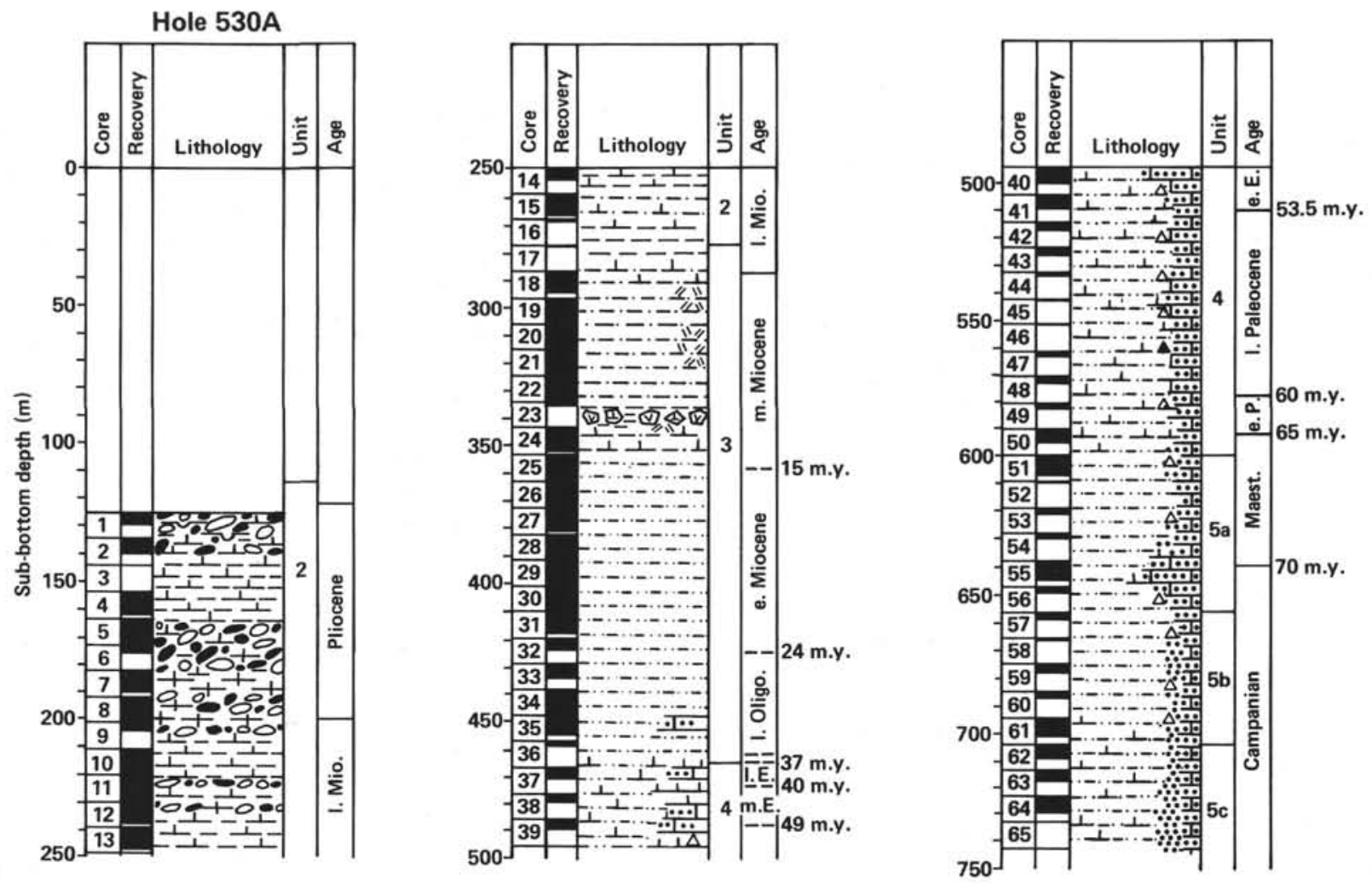

Figure 2. Lithologic units comprising the sediments and rocks encountered in Holes 530A and 530B in the Angola Basin. (Revisions of stratigraphic ages are given in Steinmetz et al., this volume.)

in foraminifer abundance records deepening of the CCD during the Quaternary. Pleistocene and Holocene sediments rich in both diatoms and calcareous microfossils are interbedded with debris-flow deposits and diatomrich mud turbidites, both largely derived from Walvis Ridge.

\section{Site 532-Walvis Ridge}

Site 532 is located on the eastern part of the Walvis Ridge close to Site 362 in a trough with relatively thick sediment fill and near the Walvis Bay zone of upwelling and high productivity. The sediments are made up of calcareous and siliceous biogenic materials with variable amounts of terrigenous clay and are extensively bioturbated. They accumulated very rapidly at rates of between 25 and $60 \mathrm{~m} / \mathrm{m}$.y. The sequences at Holes 532 and 532B are summarized in Figure 3 and are detailed in the Site 532 summary (this volume). Clearly defined fluctuations of light and dark sediment occur often over 1 to 3 $\mathrm{m}$ intervals representing time spans of 30,000 to 50,000 years. There is little evidence that the light-dark cycles were caused by carbonate dissolution within the sediment.

Only one lithologic unit was found in Holes 532 and 532B; this consists of diatom and nannofossil ooze, marl, and clay. The unit is subdivided into three subunits based on the relative proportions of biogenic components and clay. Site 532 sediments are Pleistocene to late Miocene in age.
The dominant lithologies in Subunit 1a are nannoforaminiferal and foram-nannofossil ooze and marl. Some of these have a significant diatom component. The colors are various hues of yellowish-brown and greenish-olive. The darker layers are richer in organic carbon, clay, and pyrite, but do not appear to be systematically related to any particular biogenic component. All of the sediments are thoroughly bioturbated, and the gradational contacts between light and dark layers are usually extensively burrowed.

Subunit $1 \mathrm{~b}$ is more siliceous than Subunit 1a. The dominant lithologies are nannofossil diatom smarl and sarl (see Site 530 summary). There are also minor siliceous ooze and nannofossil ooze and marl. The colors are the same hues of yellowish-brown and greenish-olive as found in Subunit 1a. Lighter and darker layers alternate. The dark layers tend to be more clay and organicrich and to contain less carbonate than the lighter layers. Sediments are highly bioturbated and burrowed.

Subunit 1c comprises a thick monotonous sequence of nannofossil marl. The colors are as for the overlying subunits, although in the bottom two-thirds the yellowish-brown colors disappear, and the dominant colors are pale and dark greenish-olive. In the bottom few cores, the colors are very light, with a slight bluish-gray tint. The dark-light interbedding is now more clearly a result of a more clay and organic-rich (dark) and a more nannofossil-rich (light) composition. There is an overall tendency toward less frequent and less intense dark layers with depth. 

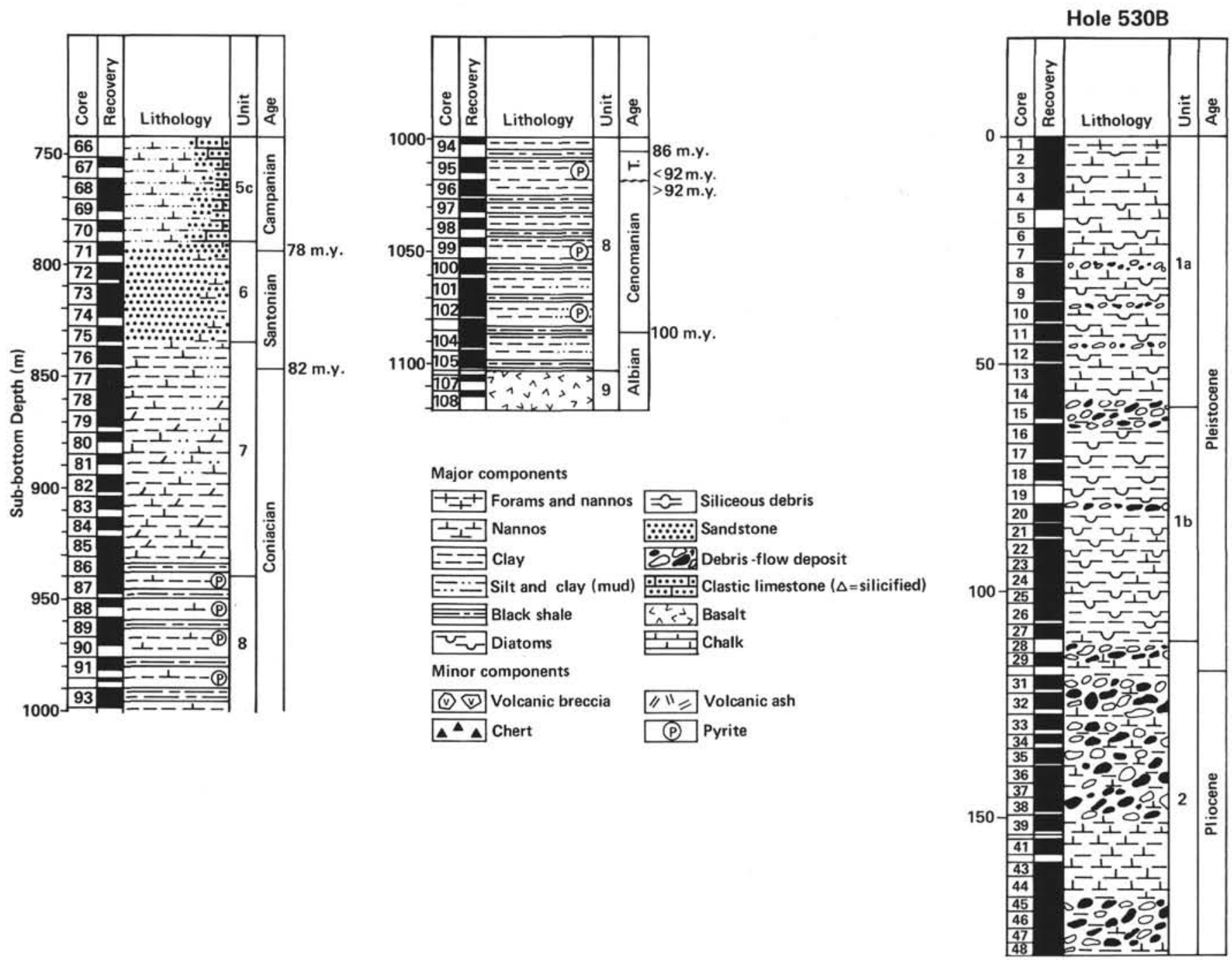

Figure 2. (Continued).

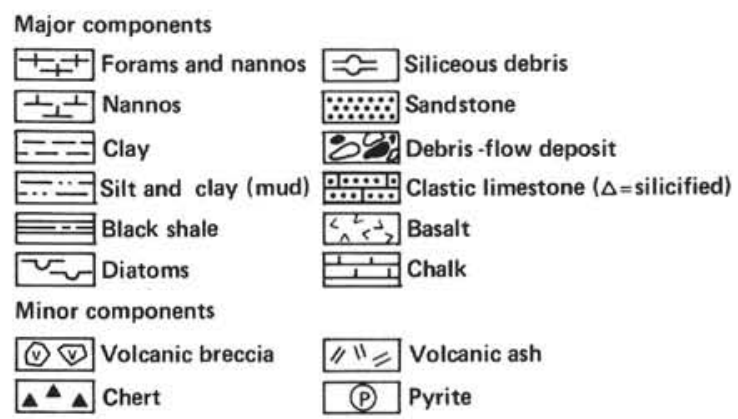

\section{RESULTS}

\section{Site 530-Angola Basin}

Through most of the sedimentary succession, organic matter content was generally low, with the important exceptions of mid-Cretaceous and Neogene deposits. The overall pattern of sediment total organic carbon (TOC) concentrations is shown in Figure 4. The different lithologic units are also indicated. TOC values of individual samples are listed in Table 2, along with their ages, sub-bottom depths, and lithologic descriptions, their atomic $\mathrm{C} / \mathrm{N}$ ratios, and their calcium carbonate contents.

Black shales within the Albian to Santonian sequences in the lower $200 \mathrm{~m}$ of Hole $530 \mathrm{~A}$ contain up to $16.5 \%$ TOC. These organic-carbon-rich layers were generally thin and were interbedded with thicker green and red claystones generally containing less than $0.5 \%$ TOC.

Organic-carbon-rich sediments in the upper $250 \mathrm{~m}$ at Site 530 have a TOC range of 1 to $7 \%$, which is smaller than in the Cretaceous sequences. These sediments are late Miocene to Holocene in age and consist of biogenic debris flows and turbidites apparently derived from

shallower-water deposits on the Walvis Ridge, where similar organic-rich sediments of the same age were recovered at Sites 362 (Foresman, 1978) and 532.

\section{Site 532-Walvis Ridge}

The content of organic matter of these sediments is high, ranging up to $9.75 \%$ dry sediment weight (Table $3)$. Organic carbon contents are consistently higher than the average of $0.2 \%$ of most pelagic sediments (Degens and Mopper, 1976), which is surprising in view of the extensive bioturbation evident in cores from these holes. The increase in TOC from about $2 \%$ in late Miocene sediments to values between 3 and $6 \%$ in Pliocene and Pleistocene oozes parallels that reported for Site 362 by Erdman and Schorno (1978) and also that found at Site 530 (Table 2 and Fig. 4).

\section{DISCUSSION}

\section{Site 530-Angola Basin}

Contents of organic carbon in sediments from the upper $300 \mathrm{~m}$ and rocks from the bottom $200 \mathrm{~m}$ at Site 530 are much higher than typical marine deposits. Waples 


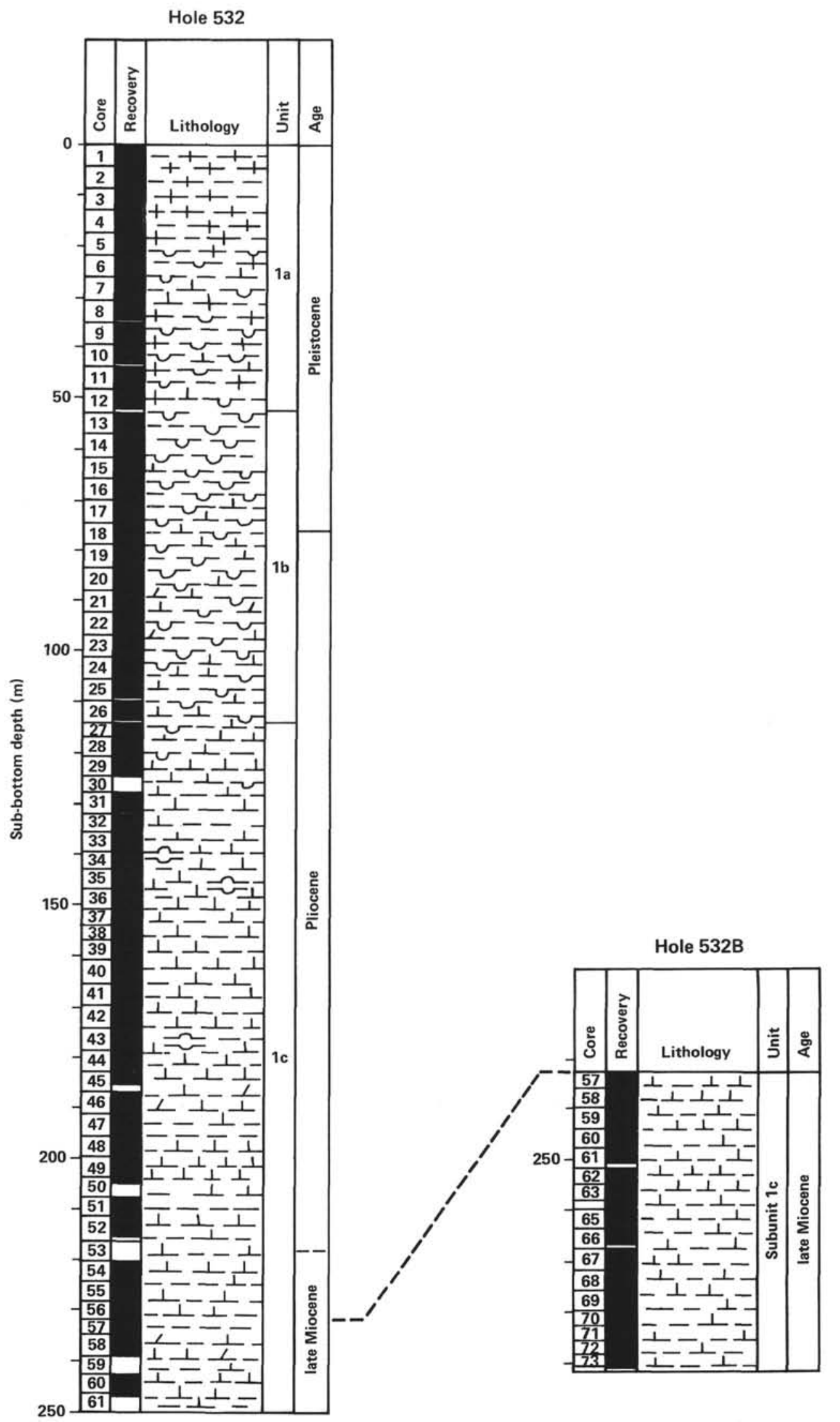

Figure 3. Lithologic subunits comprising the biogenic sediments found in Holes 532 and 532B on the Walvis Ridge. (Revisions of stratigraphic ages are given in Steinmetz et al., this volume.) 


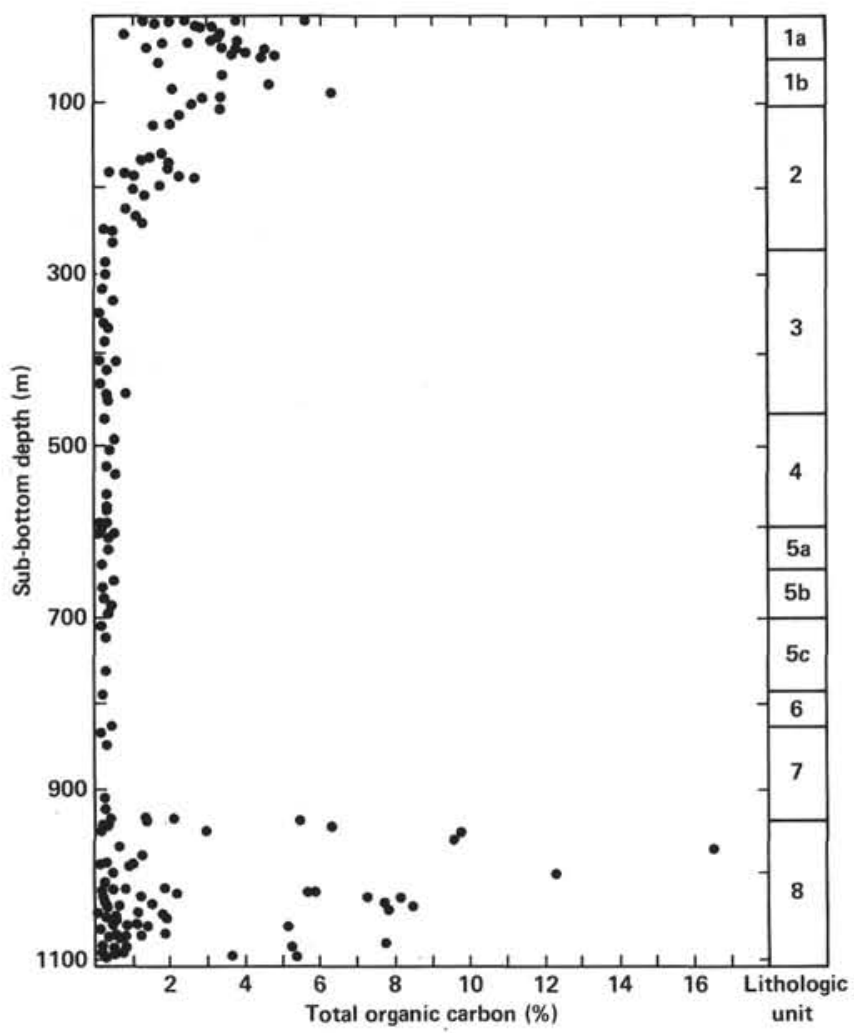

Figure 4. Total organic carbon contents expressed as percent of dry sediment weight from Site 530 in the Angola Basin. (Lithologic units are shown in Fig. 2 and described briefly in the text and fully in Site 530 summary, this volume.)

and Sloan (1980) present data from DSDP Leg 58 showing that, under uniform conditions of organic matter production and sedimentation, postdepositional degradation will decrease organic carbon concentrations to ca. $0.1 \%$ in 2 to $5 \mathrm{~m} . \mathrm{y}$. This occurs regardless of burial depth, although higher sedimentation rates appear to retard degradation.

The existence of the organic-rich zones in Holes 530A and 530B indicates significant changes in those factors important to organic matter accumulation in sediments. Table 4 summarizes average organic carbon concentrations and $\mathrm{C} / \mathrm{N}$ ratios of the samples listed in Table 2 and groups these data according to Site 530 lithologic units. The phytoplankton paleoproductivity of each unit is estimated from sedimentation rates and organic carbon contents using the expression derived by Müller and Suess (1979):

$$
\text { Paleoproductivity, } R=\frac{C \cdot P_{S}(1-\phi)}{(0.0030) S^{0.30}}
$$

where $C=$ weight percent organic carbon, $P_{s}=$ sediment density $\left(2.6 \mathrm{~g} / \mathrm{cm}^{3}\right.$ assumed here), $\phi=$ sediment porosity ( 0.9 assumed here), and $S=$ linear sedimentation rate.

Unit 8 consists of Late Cretaceous (Albian to Coniacian) black shales and red and green claystones. The average sedimentation rate of this unit is $9 \mathrm{~m} / 10^{6} \mathrm{y}$., which is five to ten times greater than the average rate for clays in deep ocean basins (Heezen and Hollister, 1971, p. 118) and reflects downslope input of material from the continental margin. Red and green claystones are low in organic carbon, averaging $0.42 \%$, whereas the black shales are rich in organic carbon. The estimated primary production rate which formed the organic matter contained within the red and green layers is $38 \mathrm{~g} \mathrm{C} / \mathrm{m}^{2} / \mathrm{y}$. According to Menzel (1974), this value is midway between the mean productivities of oligotrophic openocean subtropical areas and open-ocean edges of equatorial convergences. However, Site 530 has always been close to the African continental margin and was between $30^{\circ}$ to $35^{\circ} \mathrm{S}$ during the Late Cretaceous (Firstbrook et al., 1979). Furthermore, the method developed by Müller and Suess (1979) to estimate paleoproductivity is based upon measurements from young sediments and does not provide for the diagenetic losses of organic matter documented by Waples and Sloan (1980). For these reasons, the value of $38 \mathrm{~g} \mathrm{C} / \mathrm{m}^{2} / \mathrm{y}$. for the red and green claystones is probably low, since it is calculated from some unknown fraction of the organic matter deposited originally.

The estimated paleoproductivity leading to the organic-carbon-rich black layers is $480 \mathrm{~g} \mathrm{C} / \mathrm{m}^{2} / \mathrm{y}$. (Table 4). This is quite high and is greater than the present-day rates in upwelling areas off Mauritania $\left(250 \mathrm{~g} \mathrm{C} / \mathrm{m}^{2} / \mathrm{y}\right.$.) and off Peru $\left(300 \mathrm{~g} \mathrm{C} / \mathrm{m}^{2} / \mathrm{y}\right.$.), which are among those cited by Müller and Suess (1979) to derive their relationship between primary productivity, surface sediment organic content, and sedimentation rates. The high organic content in the black shales may have resulted from exceptional preservation of organic matter, and so this productivity estimate may be high.

Sediment accumulation rates have a profound effect upon organic matter preservation. Müller and Suess (1979) determined that only $0.01 \%$ of the primary organic matter production avoids destruction in sediments accumulating at 2 to $6 \mathrm{~mm} / 10^{3} \mathrm{y}$. The amount increases to 0.1 to $2 \%$ at accumulation rates of 2 to $13 \mathrm{~cm} / 10^{3} \mathrm{y}$. and jumps to 11 to $18 \%$ at 66 to $140 \mathrm{~cm} / 10^{3} \mathrm{y}$. Although the mean sedimentation rate in Unit 8 is $9 \mathrm{~m} / 10^{6} \mathrm{y}$. ( 9 $\mathrm{mm} / 10^{3} \mathrm{y}$.), this unit consists of a mixture of pelagic and turbiditic sediments, so organic-carbon-rich layers may represent times of rapid sedimentation as a result of turbidite deposition in addition to periods of high productivity. Alternatively, the black shales may consist of organic-carbon-rich sediments originally deposited upslope and transported into the Angola Basin by turbidity flow and slumping.

An additional factor which would lead to increased preservation of organic carbon is anoxic near-bottom waters in the Angola Basin during the Late Cretaceous. Tissot et al. (1979) and Arthur and Natland (1979) propose that, because the North and the South Atlantic were not interconnected until the Turonian (87-91 m.y. ago) and because of the shallow Walvis Ridge-Rio Grande Rise sill depth, deep-water circulation was sluggish in the Angola Basin, resulting in widespread anoxic conditions. Such conditions could have been similar to those in the modern-day Black Sea, or they could have taken the form of an expanded and intensified midwater 
Table 2. Description and organic content of sediment samples from DSDP Site 530 in the Angola Basin. Shore-based determinations are distinguished by parentheses from those done aboard ship.

\begin{tabular}{|c|c|c|c|c|c|c|}
\hline $\begin{array}{c}\text { Sample } \\
\text { (interval in } \mathrm{cm} \text { ) }\end{array}$ & $\begin{array}{l}\text { Geologic } \\
\text { age }\end{array}$ & $\begin{array}{l}\text { Sub-bottom } \\
\text { depth } \\
\text { (m) }\end{array}$ & $\begin{array}{l}\text { Lithologic } \\
\text { description }\end{array}$ & $\begin{array}{l}\mathrm{CaCO}_{3} \\
\text { (wt. \%) }\end{array}$ & $\begin{array}{l}\text { TOC } \\
\text { (wt.\%) }\end{array}$ & $\begin{array}{l}\text { Atomic } \\
\mathrm{C} / \mathrm{N}\end{array}$ \\
\hline $530 \mathrm{~B}-1-1,46-47$ & Pleistocene & 0.5 & Diatom nannofossil ooze & 62 & 1.33 & 15.2 \\
\hline $530 \mathrm{~B}-1-2,52-53$ & Pleistocene & 2.0 & Diatom nannofossil marl & 36 & 2.41 & 10.4 \\
\hline $530 \mathrm{~B}-2-1,70-71$ & Pleistocene & 3.1 & Diatom nannofossil marl & 31 & 3.78 & 11.7 \\
\hline 530B-2-2, 55-58 & Pleistocene & 4.5 & Diatom nannofossil ooze & (4) & (5.64) & (14.3) \\
\hline $530 \mathrm{~B}-2-3,70-71$ & Pleistocene & 6.1 & Diatom nannofossil ooze & 55 & 1.99 & 17.5 \\
\hline 530B-3-1, 85-86 & Pleistocene & 7.7 & Diatom nannofossil marl & 24 & & \\
\hline $530 \mathrm{~B}-3-2,85-86$ & Pleistocene & 9.2 & Diatom nannofossil marl & 34 & 1.64 & 12.0 \\
\hline $530 \mathrm{~B}-4-1,75-80$ & Pleistocene & 12.0 & Diatom nannofossil ooze & (6) & & (15.6) \\
\hline $530 \mathrm{~B}-4-1,85-86$ & Pleistocene & 12.1 & Diatom nannofossil marl & 14 & 2.77 & 13.1 \\
\hline $530 \mathrm{~B}-4-3,110-111$ & Pleistocene & 15.3 & Diatom nannofossil marl & 32 & 3.10 & 13.8 \\
\hline $530 \mathrm{~B}-6-1,105-106$ & Pleistocene & 21.1 & Diatom nannofossil marl & 23 & 3.27 & 13.7 \\
\hline $530 \mathrm{~B}-7-2,50-51$ & Pleistocene & 25.0 & Diatom nannofossil marl & 20 & 0.79 & 13.7 \\
\hline $530 \mathrm{~B}-7-3,20-21$ & Pleistocene & 26.6 & Diatom nannofossil marl & 31 & 3.31 & 14.4 \\
\hline $530 \mathrm{~B}-8-2,71-72$ & Pleistocene & 30.0 & Diatom nannofossil ooze & 11 & 3.21 & 14.4 \\
\hline 530B-9-1, 66-67 & Pleistocene & 32.9 & Diatom nannofossil marl & 39 & 2.51 & 13.3 \\
\hline $530 \mathrm{~B}-9-2,110-120$ & Pleistocene & 34.8 & Diatom nannofossil ooze & (15) & (1.79) & (7.8) \\
\hline 530B-9-3, 14-15 & Pleistocene & 35.3 & Diatom nannofossil marl & 24 & 3.85 & 13.4 \\
\hline 530B-10-1, 87-88 & Pleistocene & 37.5 & Olive diatom nannofossil marl & 21 & 3.43 & 13.6 \\
\hline $530 \mathrm{~B}-10-1,117-124$ & Pleistocene & 37.8 & Olive diatom nannofossil marl & (45) & (1.51) & (8.3) \\
\hline $530 \mathrm{~B}-10-3,74-75$ & Pleistocene & 40.3 & Dark olive diatom clay & 6 & 3.82 & 13.9 \\
\hline $530 \mathrm{~B}-11-1,70-71$ & Pleistocene & 41.7 & Olive diatom clay & 13 & 3.97 & 13.9 \\
\hline $530 \mathrm{~B}-11-2,61-62$ & Pleistocene & 43.1 & Dark olive diatom ooze & 7 & 4.54 & 13.8 \\
\hline $530 \mathrm{~B}-11-3,30-31$ & Pleistocene & 44.3 & Dark olive diatom ooze & 4 & 3.69 & 13.8 \\
\hline $530 \mathrm{~B}-12-2,78-80$ & Pleistocene & 47.7 & Olive diatom ooze & 7 & 4.53 & 13.9 \\
\hline $530 \mathrm{~B}-12-3,29-31$ & Pleistocene & 48.7 & Olive diatom ooze & 11 & 4.81 & 13.6 \\
\hline $530 \mathrm{~B}-14-3,27-29$ & Pleistocene & 57.5 & Light brown diatom nannofossil marl & 53 & 1.67 & 12.9 \\
\hline 530B-18-1, 100-101 & Pleistocene & 72.8 & Clayey diatom ooze & $<1$ & $3.40(3.54)$ & $13.9(4.4)$ \\
\hline $530 \mathrm{~B}-20-2,4-5$ & Pleistocene & 82.1 & Dark olive clayey diatom ooze & $<1$ & $4.48(4.95)$ & $13.9(6.0)$ \\
\hline $530 \mathrm{~B}-21-2,9-10$ & Pleistocene & 86.1 & Clayey nannofossil diatom ooze & 12 & 2.10 & 11.8 \\
\hline 530B-23-1, 103-104 & Pleistocene & 93.4 & Clayey nannofossil diatom ooze & $<1$ & $6.21(6.57)$ & $14.0(5.8)$ \\
\hline 530B-25-1, 95-96 & Pleistocene & 100.8 & Dark olive diatom ooze & $<1$ & $2.86(3.35)$ & $12.4(3.8)$ \\
\hline 530B-26-2, 126-128 & Pleistocene & 105.6 & Dark olive diatom ooze & $<1$ & 2.62 & 12.1 \\
\hline $530 \mathrm{~B}-27-2,46-49$ & Pleistocene & 109.2 & Dark green nannofossil diatom ooze & 3 & 3.39 & 13.7 \\
\hline $530-2-2,52-54$ & Pleistocene & 117.2 & Green diatom clay & 5 & 2.24 & 12.3 \\
\hline $530 \mathrm{~A}-1-1,92-94$ & Pliocene & 125.9 & Olive diatom nannofossil ooze & 47 & 2.08 & 24.1 \\
\hline 530B-33-2, 118-120 & Pliocene & 129.7 & Debris flow matrix & 43 & 1.62 & 13.4 \\
\hline $530 \mathrm{~B}-43-2,112-114$ & Pliocene & 162.6 & Dark olive silt-clay & 13 & 1.80 & 12.7 \\
\hline $530 \mathrm{~B}-44-2,145-148$ & Pliocene & 164.5 & Dark olive silt-clay & 5 & 1.51 & 11.4 \\
\hline $530 \mathrm{~B}-44, \mathrm{CC}(27-30)$ & Pliocene & 167.4 & Light olive silt nannofossil clay & 9 & 1.30 & 9.8 \\
\hline $530 A-5-5,27-29$ & Pliocene & 169.3 & Olive nannofossil silty clay & 15 & 1.95 & 10.9 \\
\hline $530 \mathrm{~A}-6-2,128-130$ & Pliocene & 175.3 & Nannofossil clayey diatom ooze & 5 & 1.98 & 13.4 \\
\hline $530 \mathrm{~A}-7-1,117-120$ & Pliocene & 183.2 & Light green foram nannofossil ooze & (50) & $(0.42)$ & $(9.0)$ \\
\hline $530 A-7-2,5-8$ & Pliocene & 183.6 & Green clay & 1 & 0.84 & 11.1 \\
\hline $530 \mathrm{~A}-7-2,131-133$ & Pliocene & 184.8 & Olive nannofossil clay & (15) & $(2.18)$ & (8.9) \\
\hline $530 \mathrm{~A}-7-3,30-32$ & Pliocene & 185.3 & Olive nannofossil clay & (14) & $(0.98)$ & (5.6) \\
\hline $530 A-7-6,54-56$ & Pliocene & 190.0 & Olive nannofossil clay & 16 & 2.63 & 13.2 \\
\hline $530 \mathrm{~A}-8-5,12-14$ & Late Miocene & 197.5 & Olive silty clay & 6 & 1.76 & 9.5 \\
\hline $530 \mathrm{~A}-9-1,102-104$ & Late Miocene & 202.0 & Olive nannofossil marl & 22 & 1.02 & 11.8 \\
\hline $530 \mathrm{~A}-10-1,27-29$ & Late Miocene & 210.8 & Light green nannofossil ooze & 28 & 2.29 & 13.4 \\
\hline $530 \mathrm{~A}-11-5,95-97$ & Late Miocene & 227.0 & Olive nannofossil clay & 9 & 0.92 & 9.6 \\
\hline $530 \mathrm{~A}-12-5,52-54$ & Late Miocene & 236.0 & Olive silty clay & 5 & 1.10 & 10.3 \\
\hline $530 \mathrm{~A}-13-2,67-69$ & Late Miocene & 241.0 & Olive nannofossil ooze & 34 & 1.23 & 12.8 \\
\hline $530 \mathrm{~A}-14-1,91-93$ & Late Miocene & 249.5 & Green foram nannofossil marl & 34 & 0.30 & 8.2 \\
\hline $530 \mathrm{~A}-14-3,91-93$ & Late Miocene & 252.5 & Light green foram nannofossil ooze & 46 & 0.36 & 8.0 \\
\hline $530 \mathrm{~A}-15-4,45-47$ & Late Miocene & 263.0 & Green mud & 1 & 0.46 & 5.8 \\
\hline $530 \mathrm{~A}-18-3,42-44$ & Middle Miocene & 290.0 & Green mud & $<1$ & 0.27 & 4.4 \\
\hline $530 \mathrm{~A}-19-4,30-32$ & Middle Miocene & 301.0 & Dark green clay & $<1$ & 0.26 & 4.3 \\
\hline $530 A-21-4,24-26$ & Middle Miocene & 320.0 & Green clay & $<1$ & 0.19 & 4.2 \\
\hline $530 \mathrm{~A}-22-6,81-83$ & Middle Miocene & 333.0 & Olive black clay & $<1$ & 0.49 & 7.0 \\
\hline $530 \mathrm{~A}-24-3,75-77$ & Middle Miocene & 347.0 & Green clay & $<1$ & 0.07 & 2.1 \\
\hline $530 \mathrm{~A}-25-3,87-89$ & Early Miocene & 357.0 & Green mud & $<1$ & 0.21 & 6.1 \\
\hline $530 \mathrm{~A}-26-3,48-50$ & Early Miocene & 366.0 & Green mud & $<1$ & 0.25 & 4.8 \\
\hline $530 \mathrm{~A}-27-7,35-37$ & Early Miocene & 381.5 & Brown mud & $<1$ & 0.23 & 4.3 \\
\hline $530 \mathrm{~A}-28-1,90-92$ & Early Miocene & 382.5 & Brown mud & $<1$ & 0.22 & 4.5 \\
\hline $530 \mathrm{~A}-30-4,124-126$ & Early Miocene & 406.2 & Green mud & $<1$ & 0.53 & 10.4 \\
\hline $530 \mathrm{~A}-31-2,76-78$ & Early Miocene & 412.0 & Green mud & $<1$ & 0.26 & 5.5 \\
\hline $530 A-33-2,5-7$ & Oligocene & 430.5 & Light olive mud & $<1$ & 0.12 & 3.1 \\
\hline $530 \mathrm{~A}-34-2,49-51$ & Oligocene & 440.5 & Black mud & $<1$ & 0.79 & 11.3 \\
\hline $530 \mathrm{~A}-34-2,90-92$ & Oligocene & 441.0 & Green black mud & $<1$ & 0.22 & 4.8 \\
\hline $530 \mathrm{~A}-35-1,7-9$ & Oligocene & 448.0 & Green mud & $<1$ & 0.30 & 6.4 \\
\hline $530 \mathrm{~A}-37-3,67-69$ & Late Eocene & 470.5 & Green mudstone & $<1$ & 0.25 & 7.7 \\
\hline $530 \mathrm{~A}-40-1,42-43$ & Early Eocene & 496.0 & Dark green mudstone & 9 & 0.43 & 10.2 \\
\hline $530 \mathrm{~A}-41-1,100-101$ & Early Eocene & 506.0 & Brown mudstone & $<1$ & 0.36 & 12.4 \\
\hline $530 \mathrm{~A}-42, \mathrm{CC}$ & Late Paleocene & 524.0 & Black mudstone & 2 & 0.27 & 8.1 \\
\hline $530 \mathrm{~A}-44-2,44-45$ & Late Paleocene & 535.5 & Black mudstone & 1 & 0.50 & 7.7 \\
\hline $530 \mathrm{~A}-47-1,103-105$ & Late Paleocene & 563.0 & White clastic limestone & 11 & 0.28 & 7.8 \\
\hline $530 A-48-2,19-20$ & Late Paleocene & 573.0 & Greenish black mudstone & 5 & 0.28 & 10.1 \\
\hline $530 \mathrm{~A}-49-1,49-50$ & Early Paleocene & 581.5 & Greenish black mudstone & 12 & 0.25 & 7.7 \\
\hline $530 A-50-2,12-14$ & Early Paleocene & 592.0 & Olive mudstone & 13 & 0.19 & 7.4 \\
\hline $530 \mathrm{~A}-50-2,55-57$ & Maestrichtian & 592.6 & Olive mudstone & 44 & 0.07 & 5.3 \\
\hline $530 A-50-4,21-22$ & Maestrichtian & 595.0 & Light olive marlstone & 57 & 0.09 & 7.7 \\
\hline $530 \mathrm{~A}-51-5,119-121$ & Maestrichtian & 607.0 & Dark green mudstone & 8 & 0.35 & 6.0 \\
\hline $530 \mathrm{~A}-52-1,60-61$ & Maestrichtian & 610.0 & Green mudstone & 1 & 0.30 & 14.4 \\
\hline $530 A-53-2,70-71$ & Maestrichtian & 621.0 & Olive mudstone & 12 & 0.32 & 9.1 \\
\hline $530 \mathrm{~A}-55-3,26-27$ & Maestrichtian & 641.0 & Dark green marlstone & 29 & 0.14 & 7.6 \\
\hline $530 \mathrm{~A}-57-2,84-85$ & Maestrichtian & 659.5 & Dark green mudstone & 9 & 0.42 & 15.0 \\
\hline 530A-58-1, 89-90 & Maestrichtian & 667.5 & Green mudstone & 4 & 0.16 & 10.8 \\
\hline $530 \mathrm{~A}-59-2,24-25$ & Maestrichtian & 678.0 & Dark green mudstone & 3 & 0.18 & 13.2 \\
\hline $530 \mathrm{~A}-60-2,112-114$ & Campanian & 688.1 & Dark green marlstone & 26 & 0.24 & 15.1 \\
\hline $530 \mathrm{~A}-61-1,87-88$ & Campanian & 696.0 & Green mudstone & 6 & 0.24 & 5.4 \\
\hline $530 \mathrm{~A}-63-3,62-64$ & Campanian & 717.5 & Green mudstone & 11 & 0.11 & 8.2 \\
\hline
\end{tabular}


Table 2. (Continued).

\begin{tabular}{|c|c|c|c|c|c|c|}
\hline $\begin{array}{c}\text { Sample } \\
\text { (interval in } \mathrm{cm} \text { ) }\end{array}$ & $\begin{array}{l}\text { Geologic } \\
\text { age }\end{array}$ & $\begin{array}{l}\text { Sub-bottom } \\
\text { depth } \\
\text { (m) }\end{array}$ & $\begin{array}{l}\text { Lithologic } \\
\text { description }\end{array}$ & $\begin{array}{l}\mathrm{CaCO}_{3} \\
\text { (wt. } \% \text { ) }\end{array}$ & $\begin{array}{l}\text { TOC } \\
\text { (wt.\%) }\end{array}$ & $\begin{array}{l}\text { Atomic } \\
\mathrm{C} / \mathrm{N}\end{array}$ \\
\hline $530 \mathrm{~A}-64-1,53-54$ & Campanian & 724.0 & Light olive marlstone & 24 & 0.22 & 9.1 \\
\hline $530 \mathrm{~A}-68-2,95-96$ & Campanian & 764.0 & Dark olive mudstone & $<1$ & 0.26 & 15.2 \\
\hline $530 \mathrm{~A}-71-2,50-52$ & Campanian & 792.0 & Greenish gray mudstone & 19 & 0.15 & 15.5 \\
\hline $530 \mathrm{~A}-75-4,22-23$ & Campanian & 829.5 & Green claystone & 1 & 0.37 & 21.2 \\
\hline $530 A-76-1,70-71$ & Campanian & 838.0 & Blue claystone & 1 & 0.15 & 15.3 \\
\hline $530 \mathrm{~A}-77-3,134-135$ & Campanian & 851.5 & Purple claystone & 23 & 0.24 & 17.3 \\
\hline $530 \mathrm{~A}-84-2,117-118$ & Santonian & 915.5 & Green dolomite & 41 & 0.19 & 13.7 \\
\hline $530 A-85-2,6-7$ & Santonian & 923.5 & Dark red claystone & 8 & 0.21 & 9.0 \\
\hline $530 \mathrm{~A}-86-4,145-147$ & Santonian & 937.0 & Green claystone & 4 & 0.28 & 14.6 \\
\hline $530 \mathrm{~A}-86-5,31-32$ & Santonian & 937.5 & Black claystone & $<1$ & 1.28 & 20.6 \\
\hline $530 \mathrm{~A}-86-5,33-34$ & Santonian & 937.5 & Black claystone & $<1$ & 2.02 & 21.3 \\
\hline $530 \mathrm{~A}-86-5,36-37$ & Santonian & 937.6 & Green claystone & $<1$ & 0.25 & 15.9 \\
\hline $530 \mathrm{~A}-87-1,37-38$ & Coniacian & 940.5 & Black shale & $<1$ & 5.37 & 24.0 \\
\hline $530 \mathrm{~A}-87-1,83-84$ & Coniacian & 941.0 & Black claystone & $<1$ & 1.31 & 20.2 \\
\hline $530 \mathrm{~A}-87-3,83-85$ & Coniacian & 944.0 & Dark red claystone & $<1$ & 0.25 & 9.4 \\
\hline $530 \mathrm{~A}-87-4,105-106$ & Coniacian & 945.6 & Black shale & $<1$ & 6.22 & 23.2 \\
\hline $530 \mathrm{~A}-87-4,118-119$ & Coniacian & 945.7 & Green claystone & $<1$ & 0.16 & 7.3 \\
\hline $530 \mathrm{~A}-88-1,65-66$ & Coniacian & 949.7 & Light brown limestone & 78 & 0.11 & 13.4 \\
\hline $530 \mathrm{~A}-88-3,33-34$ & Coniacian & 952.3 & Black shale & $<1$ & 9.70 & 24.7 \\
\hline $530 \mathrm{~A}-88-3,90-91$ & Coniacian & 952.9 & Black shale & $<1$ & 2.84 & 24.9 \\
\hline $530 \mathrm{~A}-89-1,34-35$ & Coniacian & 958.3 & Black shale & 15 & 9.60 & 28.9 \\
\hline $530 \mathrm{~A}-90-3,86-87$ & Coniacian & 970.9 & Black shale & 3 & 16.50 & 36.5 \\
\hline $530 \mathrm{~A}-90-3,99-100$ & Coniacian & 971.0 & Gray marlstone & 11 & 0.62 & 18.6 \\
\hline $530 \mathrm{~A}-91-4,45-46$ & Coniacian & 981.0 & Green claystone & $<1$ & 1.19 & 15.9 \\
\hline 530A-93-1, 40-41 & Coniacian & 990.4 & Green claystone & $<1$ & 0.95 & 14.1 \\
\hline 530A-93-2, 35-36 & Coniacian & 991.8 & Green shale & $<1$ & 0.18 & 10.1 \\
\hline $530 \mathrm{~A}-93-3,45-46$ & Coniacian & 993.5 & Red claystone & $<1$ & 0.11 & 7.0 \\
\hline 530A-93-3, 83-84 & Coniacian & 993.8 & Green claystone & 4 & 0.83 & 32.5 \\
\hline 530A-94-1, 42-43 & Cenomanian & 999.4 & Black shale & $<1$ & $12.27(12.66)$ & $34.5(27.8)$ \\
\hline $530 \mathrm{~A}-94-2,11-12$ & Cenomanian & 1000.6 & Dark green shale & $<1$ & 0.35 & 18.6 \\
\hline $530 \mathrm{~A}-95-4,101-102$ & Cenomanian & 1013.5 & Green claystone & $<1$ & 0.22 & 6.0 \\
\hline $530 \mathrm{~A}-95-5,0-21$ & Cenomanian & 1014.1 & Green claystone & $<1$ & $(0.20)$ & (4.5) \\
\hline $530 \mathrm{~A}-95-5,38-50$ & Cenomanian & 1014.4 & Black shale & $<1$ & (12.95) & $(36.0)$ \\
\hline $530 A-96-2,118-119$ & Cenomanian & 1019.7 & Dark red claystone & 34 & 0.11 & 10.8 \\
\hline 530A-96-3, 97-98 & Cenomanian & 1021.0 & Light brown marlstone & 10 & $(0.76)$ & (6.5) \\
\hline $530 \mathrm{~A}-96-4,29-30$ & Cenomanian & 1021.8 & Black shale & $<1$ & 1.79 & $25.9^{\circ}$ \\
\hline $530 \mathrm{~A}-96-4,40-41$ & Cenomanian & 1021.9 & Green shale & 9 & $0.39(0.41)$ & $28.4(27.0)$ \\
\hline $530 \mathrm{~A}-96-4,61-62$ & Cenomanian & 1022.1 & Black shale & $<1$ & $5.82(5.70)$ & $26.4(10.5)$ \\
\hline $530 \mathrm{~A}-96-6,88-89$ & Cenomanian & 1025.4 & Black shale & $<1$ & 2.10 & 27.9 \\
\hline 530 A-97-1, 27-29 & Cenomanian & 1026.3 & Light green limestone & 82 & $(0.15)$ & (23.8) \\
\hline $530 \mathrm{~A}-97-1,87-91$ & Cenomanian & 1026.9 & Green claystone & $<1$ & $(0.58)$ & $(6.8)$ \\
\hline 530A-97-1, 91-95 & Cenomanian & 1026.9 & Black shale & $<1$ & $(4.89)$ & (38.1) \\
\hline $530 \mathrm{~A}-97-1,99-105$ & Cenomanian & 1027.0 & Black shale & $<1$ & (10.01) & (40.3) \\
\hline $530 \mathrm{~A}-97-1,105-110$ & Cenomanian & 1027.1 & Green claystone & $<1$ & $(0.80)$ & $(5.7)$ \\
\hline $530 \mathrm{~A}-97-3,59-60$ & Cenomanian & 1029.6 & Black shale & $<1$ & $7.29(7.33)$ & $32.1(32.3)$ \\
\hline $530 \mathrm{~A}-97-3,83-84$ & Cenomanian & 1029.8 & Green claystone & $<1$ & $(1.20)$ & (6.5) \\
\hline $530 \mathrm{~A}-97-4,56-57$ & Cenomanian & 1031.1 & Black shale & $<1$ & 8.04 & 31.6 \\
\hline $530 \mathrm{~A}-97-4,87-88$ & Cenomanian & 1031.4 & Green claystone & $<1$ & 0.17 & 8.7 \\
\hline 530A-97,CC - & Cenomanian & 1035.0 & Black shale & $<1$ & 7.66 & 30.9 \\
\hline $530 A-98-2,60-61$ & Cenomanian & 1037.1 & Dark green claystone & $<1$ & 0.28 & 10.7 \\
\hline 530A-98-2, 69-70 & Cenomanian & 1037.2 & Black shale & $<1$ & 1.44 & 24.1 \\
\hline 530A-98.3, 92-93 & Cenomanian & 1038.9 & Black shale & 2 & $8.57(7.71)$ & $30.2(25.6)$ \\
\hline $530 \mathrm{~A}-98-3,110-111$ & Cenomanian & 1039.1 & Dark green claystone & $<1$ & 0.47 & 18.0 \\
\hline 530A-99-1, 107-108 & Cenomanian & 1045.1 & Green shale & $<1$ & 0.23 & 13.0 \\
\hline 530A-99-2, 67-69 & Cenomanian & 1046.2 & Light green limestone & 76 & 0.08 & 10.6 \\
\hline $530 \mathrm{~A}-99-3,118-120$ & Cenomanian & 1048.2 & Light green marlstone & 15 & $(1.04)$ & $(17.0)$ \\
\hline $530 A-99-5,70-71$ & Cenomanian & 1050.7 & Black shale & $<1$ & 1.73 & 23.3 \\
\hline 530A-99-5, 86-87 & Cenomanian & 1050.9 & Green shale & $<1$ & 0.38 & 10.2 \\
\hline $530 A-100-1,85-86$ & Cenomanian & 1053.9 & Dark green shale & $<1$ & 0.28 & 11.3 \\
\hline 530A-100-1, 99-100 & Cenomanian & 1054.0 & Black shale & $<1$ & 1.92 & 23.1 \\
\hline $530 \mathrm{~A}-100-2,120-121$ & Cenomanian & 1055.7 & Light green marlstone & 14 & $(0.41)$ & (6.9) \\
\hline $530 \mathrm{~A}-100-3,142-143$ & Cenomanian & 1057.4 & Light green marlstone & 35 & $(0.45)$ & (8.2) \\
\hline $530 \mathrm{~A}-100-4,114-115$ & Cenomanian & 1058.6 & Dark green marlstone & 29 & & \\
\hline $530 \mathrm{~A}-100-5,38-39$ & Cenomanian & 1059.4 & Dark red claystone & $<1$ & $(0.38)$ & $(0.9)$ \\
\hline 530A-101-1, 16-17 & Cenomanian & 1062.2 & Green claystone & $<1$ & $(0.70)$ & (1.6) \\
\hline $530 \mathrm{~A}-101-2,8-9$ & Cenomanian & 1063.6 & Black shale & $<1$ & 0.93 & 14.2 \\
\hline $530 A-101-2,17-18$ & Cenomanian & 1063.7 & Light green marlstone & 26 & 0.48 & 17.2 \\
\hline $530 \mathrm{~A}-101-2,21-22$ & Cenomanian & 1063.7 & Black shale & $<1$ & 5.08 & 28.5 \\
\hline $530 A-101-4,36-37$ & Cenomanian & 1066.9 & Dark green claystone & 21 & 1.31 & 37.1 \\
\hline $530 \mathrm{~A}-101-4,55-56$ & Cenomanian & 1067.1 & Light green marlstone & 40 & 0.08 & 10.4 \\
\hline $530 A-101-6,86-87$ & Cenomanian & 1070.4 & Light green marlstone & 16 & $(0.38)$ & $(0.7)$ \\
\hline $530 \mathrm{~A}-101-7,49-50$ & Cenomanian & 1071.5 & Light green claystone & 5 & $(0.30)$ & $(0.8)$ \\
\hline $530 \mathrm{~A}-102-1,149-150$ & Cenomanian & 1072.5 & Dark green claystone & $<1$ & 1.16 & 18.5 \\
\hline $530 \mathrm{~A}-102-3,120-121$ & Cenomanian & 1075.2 & Black shale & $<1$ & 1.73 & 22.8 \\
\hline $530 \mathrm{~A}-102-3,130-131$ & Cenomanian & 1075.3 & Dark green claystone & 11 & 0.45 & 20.4 \\
\hline $530 \mathrm{~A}-102-3,145-146$ & Cenomanian & 1075.5 & Light green marlstone & 34 & $(0.49)$ & (9.6) \\
\hline $530 \mathrm{~A}-103-4,85-86$ & Cenomanian & 1083.4 & Olive black mudstone & $<1$ & 7.70 & 30.2 \\
\hline $530 \mathrm{~A}-103-6,22-23$ & Cenomanian & 1085.7 & Green claystone & $<1$ & $0.16(0.33)$ & $10.5(2.8)$ \\
\hline $530 \mathrm{~A}-104-1,124-125$ & Albian & 1086.2 & Light green marlstone & 55 & $(0.71)$ & (7.4) \\
\hline $530 \mathrm{~A}-104-2,141-142$ & Albian & 1087.9 & Dark green claystone & 3 & 0.41 & 11.7 \\
\hline $530 \mathrm{~A}-104-3,0-1$ & Albian & 1088.0 & Black claystone & $<1$ & 5.21 & 29.0 \\
\hline $530 A-104-4,91-92$ & Albian & 1090.4 & Green claystone & $<1$ & 0.47 & 17.1 \\
\hline $530 \mathrm{~A}-104-4,123-124$ & Albian & 1090.7 & Dark green claystone & $<1$ & 0.18 & 11.3 \\
\hline $530 \mathrm{~A}-104-5,61-62$ & Albian & 1091.6 & Black claystone & 3 & 0.57 & 23.1 \\
\hline $530 A-105-1,23-24$ & Albian & 1094.2 & Dark green claystone & $<1$ & 0.23 & 12.4 \\
\hline $530 \mathrm{~A}-105-2,139-140$ & Albian & 1096.9 & Black shale & $<1$ & 5.28 & 28.2 \\
\hline $530 \mathrm{~A}-105-3,18-20$ & Albian & 1097.2 & Light green limestone & 64 & $(0.44)$ & (8.1) \\
\hline $530 \mathrm{~A}-105-4,9-10$ & Albian & 1098.6 & Black shale & 6 & 3.60 & 30.0 \\
\hline $530 \mathrm{~A}-105-4,35-36$ & Albian & 1098.9 & Dark green claystone & $<1$ & $0.16(0.36)$ & $9.4(3.1)$ \\
\hline $530 \mathrm{~A}-105, \mathrm{CC} 3-4$ & Albian & 1103.0 & Red claystone & $<1$ & 0.15 & 8.3 \\
\hline
\end{tabular}


Table 3. Descriptions and organic contents of sediment samples from DSDP Site 532 on the Walvis Ridge. Shore-based determinations are distinguished by parentheses from those done aboard ship.

\begin{tabular}{|c|c|c|c|c|c|c|}
\hline $\begin{array}{c}\text { Sample } \\
\text { (interval in } \mathrm{cm} \text { ) }\end{array}$ & $\begin{array}{l}\text { Geologic } \\
\text { age }\end{array}$ & $\begin{array}{l}\text { Sub-bottom } \\
\text { depth } \\
\text { (m) }\end{array}$ & $\begin{array}{l}\text { Lithologic } \\
\text { description }\end{array}$ & $\begin{array}{l}\mathrm{CaCO}_{3} \\
\text { (wt.\%) }\end{array}$ & $\begin{array}{c}\text { TOC } \\
\text { (wt. \%) }\end{array}$ & $\begin{array}{c}\text { Atomic } \\
\mathrm{C} / \mathrm{N}\end{array}$ \\
\hline $532-1-2,7-9$ & Pleistocene & 1.6 & Light olive nannofossil foram ooze & 68 & 3.66 & 12.1 \\
\hline $532-2-2,80-90$ & Pleistocene & 6.3 & Olive nannofossil foram marl & (38) & (3.74) & (14.3) \\
\hline $532-2-3,6-8$ & Pleistocene & 7.1 & Dark olive nannofossil foram marl & 38 & $(2.70)$ & - \\
\hline $532-2-3,60-62$ & Pleistocene & 7.6 & Light olive nannofossil foram ooze & 62 & $(1.42)$ & - \\
\hline $532-3-2,29-31$ & Pleistocene & 10.2 & Light olive nannofossil foram ooze & 73 & $(0.99)$ & - \\
\hline $532-3-3,106-108$ & Pleistocene & 11.4 & Olive nannofossil foram ooze & 58 & 3.28 & 14.7 \\
\hline $532-4-2,17-19$ & Pleistocene & 14.5 & Light olive nannofossil foram ooze & 68 & (1.83) & - \\
\hline $532-4, C \mathrm{C}$ & Pleistocene & 17.2 & Olive nannofossil foram ooze & 40 & $4.80(4.88)$ & $14.8(5.3)$ \\
\hline $532-5-1,96-98$ & Pleistocene & 18.2 & Olive nannofossil foram ooze & 41 & (5.64) & (17.0) \\
\hline $532-5-2,100-110$ & Pleistocene & 19.7 & Dark olive smarl & (4) & (9.75) & (26.3) \\
\hline $532-5-3,96-98$ & Pleistocene & 21.2 & Light olive diatom foram ooze & 70 & 1.98 & 15.2 \\
\hline $532-6-2,55-56$ & Pleistocene & 23.7 & Dark olive foram nannofossil marl & 34 & $(6.06)$ & (7.4) \\
\hline $532-6-2,144-145$ & Pleistocene & 24.5 & Light olive foram nannofossil ooze & 62 & (2.26) (2.78) & (14.8) \\
\hline $532-7-1,75-76$ & Pleistocene & 26.8 & Light olive diatom foram marl & 40 & (2.53) & (20.4) \\
\hline $532-8-2,70-71$ & Pleistocene & 32.6 & Light olive diatom foram marl & 47 & 1.70 & 13.2 \\
\hline $532-8-2,100-101$ & Pleistocene & 32.9 & Light olive diatom foram marl & 39 & 2.93 & 14.7 \\
\hline $532-8-2,145-146$ & Pleistocene & 33.4 & Olive foram nannofossil marl & 41 & 3.64 & 15.0 \\
\hline $532-8-3,20-30$ & Pleistocene & 33.6 & Dark olive foram nannofossil marl & (12) & $(5.41)$ & (15.9) \\
\hline $532-9-1,16-18$ & Pleistocene & 35.0 & Dark olive foram nannofossil ooze & 62 & 2.01 & 13.5 \\
\hline $532-9-2,108-110$ & Pleistocene & 37.4 & Light olive foram nannofossil marl & 42 & 3.57 & 14.9 \\
\hline $532-9-3,123-124$ & Pleistocene & 39.0 & Light olive foram nannofossil ooze & 58 & 1.16 & 12.0 \\
\hline $532-10-2,64-66$ & Pleistocene & 41.3 & Light olive nannofossil ooze & 58 & 1.26 & 12.4 \\
\hline $532-10-2,105-107$ & Pleistocene & 41.8 & Light olive nannofossil marl & 50 & $(2.20)$ & (4.8) \\
\hline $532-10-3,44-45$ & Pleistocene & 42.6 & Olive foram nannofossil marl & 50 & (3.11) & (12.0) \\
\hline $532-10, C C(9-11)$ & Pleistocene & 43.6 & Dark olive nannofossil diatom ooze & 23 & 4.30 & 14.9 \\
\hline $532-11-2,69-70$ & Pleistocene & 45.8 & Light olive foram nannofossil ooze & 53 & $(1.80)$ & - \\
\hline $532-11-3,10-20$ & Pleistocene & 46.7 & Dark olive diatom nannofossil marl & (11) & (6.51) & (20.0) \\
\hline $532-11-3,53-54$ & Pleistocene & 47.1 & Light olive diatom foram ooze & 41 & 5.52 & 15.9 \\
\hline $532-11-3,149-150$ & Pleistocene & 48.0 & Dark olive foram nannofossil ooze & 55 & 1.88 & 13.3 \\
\hline $532-12-1,79-80$ & Pleistocene & 48.8 & Light olive diatom foram ooze & 42 & (2.95) & (11.9) \\
\hline $532-12-2,79-80$ & Pleistocene & 50.3 & Dark olive diatom nannofossil marl & 33 & $(5.04)$ & (13.9) \\
\hline $532-13-1,69-71$ & Pleistocene & 53.1 & Dark olive diatom nannofossil marl & 25 & 3.48 & 14.8 \\
\hline $532-13-2,53-56$ & Pleistocene & 54.4 & Dark olive diatom nannofossil marl & 27 & 2.74 & 13.5 \\
\hline $532-13-3,89-92$ & Pleistocene & 56.3 & Light olive diatom nannofossil marl & 41 & (2.65) & (4.7) \\
\hline $532-13, \mathrm{CC}(13-15)$ & Pleistocene & 56.8 & Light olive diatom nannofossil marl & 43 & $(1.52)$ & - \\
\hline $532-14-2,38-39$ & Pleistocene & 58.7 & Dark olive diatom nannofossil marl & 15 & 5.91 & 15.5 \\
\hline $532-14-2,55-65$ & Pleistocene & 58.9 & Dark olive diatom nannofossil marl & (9) & (6.18) & (15.2) \\
\hline $532-14-3,38-39$ & Pleistocene & 60.2 & Light olive diatom nannofossil marl & 44 & 2.68 & 14.8 \\
\hline $532-15-2,34-35$ & Pleistocene & 63.0 & Light olive diatom nannofossil marl & 27 & $(3.45)$ & - \\
\hline $532-15-3,34-35$ & Pleistocene & 64.5 & Light olive diatom nannofossil marl & 42 & $(2.01)$ & - \\
\hline $532-16-1,118-120$ & Pleistocene & 66.8 & Light olive nannofossil diatom ooze & 48 & $(2.46)$ & (12.7) \\
\hline $532-16-2,122-124$ & Pleistocene & 68.3 & Dark olive nannofossil diatom ooze & 19 & $(2.65)$ & (12.5) \\
\hline $532-16-3,40-42$ & Pleistocene & 69.0 & Medium olive diatom nannofossil marl & 25 & (2.97) & (14.9) \\
\hline $532-17-1,81-83$ & Pleistocene & 70.8 & Light green-gray diatom nannofossil ooze & 43 & (3.15) & (11.4) \\
\hline $532-17-2,81-83$ & Pleistocene & 72.3 & Dark olive diatom nannofossil ooze & 32 & 3.20 & 14.6 \\
\hline $532-17-3,91-93$ & Pleistocene & 73.9 & Light olive nannofossil diatom marl & 32 & $(3.06)$ & - \\
\hline $532-18-1,91-93$ & Pleistocene & 75.3 & Dark olive nannofossil diatom marl & 21 & (4.12) & (19.2) \\
\hline $532-18-2,91-93$ & Pliocene & 76.8 & Light olive nannofossil diatom marl & 20 & 4.30 & 15.3 \\
\hline $532-19-2,82-84$ & Pliocene & 81.1 & Olive diatom nannofossil marl & 39 & $(4.02)$ & (13.7) \\
\hline $532-19-3,20-30$ & Pliocene & 82.0 & Dark olive diatom nannofossil marl & (11) & $(5.02)$ & (15.2) \\
\hline $532-19-3,81-83$ & Pliocene & 82.6 & Light olive diatom nannofossil marl & 28 & (3.37) & $(12.4)$ \\
\hline $532-20-1,70-72$ & Pliocene & 83.9 & Dark olive nannofossil clay ooze & 31 & (3.15) & (15.6) \\
\hline $532-20-3,71-73$ & Pliocene & 86.9 & Light olive nannofossil diatom ooze & 31 & (4.91) & (13.2) \\
\hline $532-21-2,29-30$ & Pliocene & 89.4 & Dark olive smarl & 8 & $6.35(5.90)$ & $15.5(8.6)$ \\
\hline $532-21-3,88-90$ & Pliocene & 91.5 & Light olive smarl & 48 & $(1.36)$ & (21.7) \\
\hline $532-22-1,136-139$ & Pliocene & 93.4 & Light olive smarl & 40 & (2.56) & $(12.4)$ \\
\hline $532-22-2,136-139$ & Pliocene & 94.9 & Dark olive smarl & 41 & $4.12(4.18)$ & $21.4(21.3)$ \\
\hline $532-22-3,130-133$ & Pliocene & 96.3 & Light olive smarl & 32 & $(2.76)$ & (5.6) \\
\hline $532-23-1,59-61$ & Pliocene & 97.0 & Light olive dolomitic smarl & 45 & & \\
\hline $532-23-3,34-36$ & Pliocene & 99.7 & Light olive dolomitic smarl & 58 & (4.05) & (13.2) \\
\hline $532-24-1,109-112$ & Pliocene & 101.9 & Light olive nannofossil marl & 55 & (2.31) & (8.6) \\
\hline $532-24-2,127-130$ & Pliocene & 103.6 & Light olive nannofossil marl & 50 & 2.65 & 17.1 \\
\hline $532-24-3,93-96$ & Pliocene & 104.7 & Dark olive diatom nannofossil ooze & 23 & 3.40 & 15.0 \\
\hline $532-25-1,51-52$ & Pliocene & 105.7 & Dark olive nannofossil clay & 11 & 6.04 & 16.4 \\
\hline $532-25-2,51-52$ & Pliocene & 107.2 & Light olive nannofossil clay & 31 & 3.03 & 13.9 \\
\hline $532-25-2,80-90$ & Pliocene & 107.5 & Dark olive nannofossil clay & (16) & (6.41) & (15.9) \\
\hline $532-26-1,49-50$ & Pliocene & 110.1 & Light olive diatom nannofossil ooze & 40 & 2.47 & 15.4 \\
\hline $532-26-2,5-6$ & Pliocene & 111.2 & Light olive diatom nannofossil ooze & 45 & (1.56) & - \\
\hline $532-27-1,86-88$ & Pliocene & 114.9 & Olive diatom nannofossil marl & 32 & 3.87 & 16.2 \\
\hline $532-27-2,66-68$ & Pliocene & 116.2 & Dark olive diatom nannofossil marl & 13 & 4.48 & 14.8 \\
\hline $532-27-2,86-88$ & Pliocene & 116.4 & Light olive foram nannofossil marl & 34 & (1.99) & (12.4) \\
\hline $532-28-2,18-20$ & Pliocene & 119.1 & Olive diatom nannofossil marl & 27 & $(2.26)$ & (13.2) \\
\hline $532-29-2,10-13$ & Pliocene & 122.4 & Light olive diatom nannofossil marl & 45 & (2.51) & (11.8) \\
\hline $532-29-3,10-13$ & Pliocene & 123.9 & Dark olive diatom nannofossil marl & 25 & 4.44 & 15.2 \\
\hline $532-31-1,144-145$ & Pliocene & 128.8 & Dark olive clay & 21 & $(3.86)$ & (8.4) \\
\hline $532-31-2,64-65$ & Pliocene & 129.5 & Dark olive clay & 12 & 5.87 & 16.5 \\
\hline $532-31-2,65-75$ & Pliocene & 129.6 & Dark olive clay & (36) & (3.71) & (16.1) \\
\hline $532-31-3,18-19$ & Pliocene & 130.6 & Light olive nannofossil marl & 46 & $(2.26)$ & - \\
\hline $532-32-1,11-13$ & Pliocene & 131.9 & Dark olive silty clay & 20 & 5.86 & 16.5 \\
\hline $532-32-2,46-49$ & Pliocene & 133.8 & Light olive diatom nannofossil marl & 26 & $(1.80)$ & - \\
\hline $532-32-2,147-149$ & Pliocene & 134.8 & Light olive nannofossil marl & 62 & & \\
\hline $532-33-2,61-62$ & Pliocene & 137.3 & Dark olive nannofossil marl & 39 & (2.98) & - \\
\hline $532-33-3,41-43$ & Pliocene & 138.6 & Light olive nannofossil marl & 59 & $(0.80)$ & - \\
\hline $532-34-1,121-122$ & Pliocene & 140.4 & Light olive siliceous nannofossil marl & 53 & $(0.91)$ & $(11.4)$ \\
\hline $532-34-2,49-51$ & Pliocene & 141.2 & Dark olive siliceous nannofossil marl & 33 & (2.13) & - \\
\hline $532-34-2,71-72$ & Pliocene & 141.4 & Light olive siliceous nannofossil marl & 25 & $5.47(5.62)$ & $16.1(9.8)$ \\
\hline $532-35-2,94-96$ & Pliocene & 145.0 & Light olive siliceous nannofossil ooze & 55 & (2.17) & (9.9) \\
\hline $532-35-3,70-72$ & Pliocene & 146.3 & Dark olive nannofossil marl & 43 & 3.27 & 14.9 \\
\hline $532-36-1,71-72$ & Pliocene & 147.3 & Light olive nannofossil marl & 53 & $(2.47)$ & (13.2) \\
\hline $532-37-2,45-55$ & Pliocene & 152.6 & Dark olive nannofossil marl & (39) & $(2.31)$ & (14.5) \\
\hline $532-38-1,65-68$ & Pliocene & 154.5 & Light olive nannofossil marl & 57 & $(0.94)$ & - \\
\hline
\end{tabular}


Table 3. (Continued).

\begin{tabular}{|c|c|c|c|c|c|c|}
\hline $\begin{array}{c}\text { Sample } \\
\text { (interval in } \mathrm{cm} \text { ) }\end{array}$ & $\begin{array}{l}\text { Geologic } \\
\text { age }\end{array}$ & $\begin{array}{l}\text { Sub-bottom } \\
\text { depth } \\
\text { (m) }\end{array}$ & $\begin{array}{l}\text { Lithologic } \\
\text { description }\end{array}$ & $\begin{array}{l}\mathrm{CaCO}_{3} \\
\text { (wt.\%) }\end{array}$ & $\begin{array}{l}\text { TOC } \\
\text { (wt. \%) }\end{array}$ & $\begin{array}{l}\text { Atomic } \\
\mathrm{C} / \mathrm{N}\end{array}$ \\
\hline $532-38-2,50-53$ & Pliocene & 155.8 & Dark olive nannofossil marl & 29 & 3.12 & 15.5 \\
\hline $532-39-2,60-62$ & Pliocene & 158.9 & Dark olive nannofossil marl & 45 & (1.31) & - \\
\hline $532-39-3,60-62$ & Pliocene & 159.4 & Light olive nannofossil marl & 60 & (3.56) & $(14.1)$ \\
\hline $532-40-1,75-76$ & Pliocene & 161.6 & Dark olive nannofossil marl & 37 & 2.99 & 15.4 \\
\hline $532-41-2,80-82$ & Pliocene & 167.5 & Light olive nannofossil clay & 45 & (2.37) & (9.6) \\
\hline $532-41-3,4-5$ & Pliocene & 168.2 & Dark olive nannofossil clay & 27 & 4.11 & 16.6 \\
\hline $532-42-2,71-72$ & Pliocene & 171.8 & Light olive nannofossil marl & 58 & (1.91) & (6.0) \\
\hline $532-42-2,79-80$ & Pliocene & 171.9 & Light olive nannofossil marl & 54 & 1.54 & 14.2 \\
\hline $532-42-2,140-141$ & Pliocene & 172.5 & Dark olive nannofossil marl & 34 & 2.90 & 14.4 \\
\hline $532-43-1,71-72$ & Pliocene & 174.7 & Light olive nannofossil marl & 50 & $(1.30)(1.03)$ & (18.5) (11.2) \\
\hline $532-43-1,117-118$ & Pliocene & 175.2 & Dark olive nannofossil marl & 38 & (3.22) & (12.8) \\
\hline $532-43-1,130-140$ & Pliocene & 175.3 & Dark olive nannofossil marl & (24) & (3.92) & (13.7) \\
\hline $532-44-3,36-37$ & Pliocene & 180.8 & Dark olive nannofossil clay & 30 & (4.24) & (12.7) \\
\hline $532-44-3,110-111$ & Pliocene & 182.5 & Light olive nannofossil marl & 53 & 1.69 & 13.8 \\
\hline $532-45-1,116-119$ & Pliocene & 184.0 & Light olive nannofossil marl & 55 & $(2.20)$ & $(13.0)$ \\
\hline $532-45-2,42-45$ & Pliocene & 184.7 & Light olive nannofossil marl & 68 & $(0.55)$ & - \\
\hline $532-46-2,100-101$ & Pliocene & 189.3 & Light olive nannofossil marl & 44 & $2.44(2.04)$ & $14.7(14.7)$ \\
\hline $532-46-3,96-99$ & Pliocene & 190.8 & Light olive nannofossil marl & 55 & $(1.75)$ & $(16.1)$ \\
\hline $532-47-3,110-112$ & Pliocene & 195.3 & Dark olive nannofossil marl & 41 & (2.59) & - \\
\hline $532-48-1,64-67$ & Pliocene & 196.3 & Light olive nannofossil marl & 62 & $(0.96)(0.90)$ & $(13.3)$ \\
\hline $532-48-2,142-145$ & Pliocene & 198.5 & Dark olive nannofossil marl & 34 & $(3.75)$ & $(15.4)$ \\
\hline $532-49-1,70-71$ & Pliocene & 200.3 & Light olive nannofossil marl & 55 & (1.65) & (43.5) \\
\hline $532-49-2,86-92$ & Pliocene & 202.0 & Light olive nannofossil marl & 55 & $(0.45)$ & (9.3) \\
\hline $532-49, C C(3-4)$ & Pliocene & 203.6 & Dark olive nannofossil marl & 37 & 2.02 & 13.9 \\
\hline $532-50-1,82-83$ & Pliocene & 204.8 & Dark olive nannofossil marl & 46 & 2.24 & 14.8 \\
\hline $532-51-1,144-150$ & Pliocene & 209.1 & Light olive nannofossil marl & 61 & 2.02 & 24.7 \\
\hline $532-51-2,75-78$ & Pliocene & 209.9 & Light olive nannofossil marl & 63 & 1.03 & 14.0 \\
\hline $532-52-1,64-70$ & Pliocene & 212.3 & Light olive nannofossil marl & 44 & 2.86 & 26.2 \\
\hline $532-52-2,141-147$ & Pliocene & 214.5 & Dark olive nannofossil clay & 72 & 2.24 & 17.5 \\
\hline $532-54-1,81-87$ & Late Miocene & 220.8 & Light olive nannofossil marl & 47 & 3.95 & 30.1 \\
\hline $532-56-1,100-104$ & Late Miocene & 229.0 & Light olive nannofossil marl & 65 & 0.68 & 14.2 \\
\hline $532-56-2,34-39$ & Late Miocene & 229.9 & Dark olive nannofossil marl & 47 & 1.99 & 14.7 \\
\hline $532-57-1,109-110$ & Late Miocene & 232.5 & Dark olive nannofossil marl & 49 & 2.20 & 15.0 \\
\hline $532-58-2,67-68$ & Late Miocene & 237.0 & Dark olive nannofossil clay & 32 & 3.11 & 15.0 \\
\hline $532 \mathrm{~B}-58-2,80-90$ & Late Miocene & 237.2 & Dark olive nannofossil clay & (44) & $(2.06)$ & (16.1) \\
\hline $532 \mathrm{~B}-58-3,0-6$ & Late Miocene & 238.8 & Light olive nannofossil marl & 51 & 2.04 & 15.0 \\
\hline $532 \mathrm{~B}-59-1,80-86$ & Late Miocene & 240.6 & Light olive nannofossil marl & 45 & 1.02 & 13.0 \\
\hline 532B-59-2, 80-90 & Late Miocene & 242.1 & Light olive nannofossil marl & (35) & (1.69) & (8.9) \\
\hline 532B-59-2, 106-112 & Late Miocene & 242.3 & Dark olive nannofossil marl & 37 & 2.66 & 17.6 \\
\hline $532-60-2,42-48$ & Late Miocene & 244.8 & Light olive nannofossil marl & 48 & 1.23 & 13.9 \\
\hline $532 \mathrm{~B}-61-3,5-11$ & Late Miocene & 250.3 & Dark olive nannofossil mari & 46 & 1.66 & 18.9 \\
\hline $532 \mathrm{~B}-61, \mathrm{CC}(20-21)$ & Late Miocene & 250.8 & Light olive nannofossil marl & 50 & $1.43(1.43)$ & $13.6(13.6)$ \\
\hline $532 \mathrm{~B}-62-2,124-130$ & Late Miocene & 254.0 & Light olive nannofossil marl & 64 & 0.57 & 10.8 \\
\hline $532 \mathrm{~B}-63, \mathrm{CC}(4-5)$ & Late Miocene & 257.7 & Dark olive nannofossil clay & 29 & 2.17 & 13.9 \\
\hline 532B-64-1, 80-81 & Late Miocene & 258.5 & Light olive nannofossil ooze & 60 & (2.92) & - \\
\hline 532B-65-1, 51-54 & Late Miocene & 260.2 & Dark olive nannofossil marl & 44 & 1.84 & 14.9 \\
\hline $532 \mathrm{~B}-65-2,80-90$ & Late Miocene & 262.0 & Dark olive nannofossil marl & (51) & (1.44) & (12.2) \\
\hline 532B-65-2, 109-111 & Late Miocene & 262.3 & Light olive nannofossil marl & 59 & $(0.21)$ & (10.1) \\
\hline $532 \mathrm{~B}-66, \mathrm{CC}(7-13)$ & Late Miocene & 267.1 & Dark olive nannofossil clay & 38 & 3.27 & 14.5 \\
\hline $532 \mathrm{~B}-67-2,54-55$ & Late Miocene & 269.1 & Light olive nannofossil marl & 49 & 2.15 & 14.3 \\
\hline $532 \mathrm{~B}-67-3,54-55$ & Late Miocene & 270.6 & Light olive nannofossil marl & 69 & (2.61) & - \\
\hline $532 B-68-1,54-55$ & Late Miocene & 272.0 & Light olive nannofossil marl & 47 & $(0.68)$ & - \\
\hline $532 \mathrm{~B}-68-2,54-55$ & Late Miocene & 273.5 & Light olive nannofossil marl & 68 & (0.38) & (17.9) \\
\hline $532 \mathrm{~B}-69-2,19-20$ & Late Miocene & 277.2 & Light olive nannofossil marl & 57 & 2.11 & 20.1 \\
\hline $532 \mathrm{~B}-69-2,89-90$ & Late Miocene & 277.9 & Light olive nannofossil marl & 61 & $(2.85)$ & - \\
\hline 532B-70-2, 70-71 & Late Miocene & 281.5 & Light olive nannofossil marl & 73 & $(0.14)$ & (10.3) \\
\hline 532B-71-2, 76-82 & Late Miocene & 284.6 & Light olive nannofossil marl & 74 & $(0.50)$ & (52.6) \\
\hline $532 B-72-1,34-36$ & Late Miocene & 285.6 & Light olive nannofossil marl & 78 & $(0.98)$ & (22.7) \\
\hline $532 \mathrm{~B}-72-2,34-36$ & Late Miocene & 287.1 & Light olive nannofossil marl & 72 & $(0.10)$ & - \\
\hline $532 \mathrm{~B}-73-2,14-20$ & Late Miocene & 290.0 & Light olive nannofossil marl & 63 & $1.51(2.11)$ & $15.6(5.9)$ \\
\hline 532B-74,CC - & Late Miocene & 291.3 & Light olive nannofossil marl & 71 & $(0.50)$ & (47.6) \\
\hline
\end{tabular}

oxygen minimum zone. These situations would result in enhanced preservation of organic matter, both during sinking through the water column and after incorporation into surface sediments. However, Förster (1978) concludes from fossil ammonite distributions that the interconnection between the North and the South Atlantic oceans became established in the late Albian (ca. 102 m.y. ago), thus providing a northern source of oxygencontaining water to the Angola Basin and lessening the likelihood of strongly developed anoxic conditions during the Late Cretaceous.

Two features present in Unit 8 argue against the existence of a Black Sea type of strongly anoxic condition. First, burrowing by benthic animals is plainly present in most parts of the unit (Hay et al., 1982; Site 530 summary, this volume). Second, black shales are interbedded among green and red claystones (Table 2, Fig. 5).
The red layers make up about $44 \%$ of the unit and evidently were deposited under oxygenated bottom waters. These two observations preclude the existence of permanently anoxic bottom waters in the Late Cretaceous Angola Basin, but do not rule out short periods of bottom water anoxia or the presence of a midwater oxygendepleted layer.

Layers of green claystones make up about $47 \%$ of Unit 8 and are the type of organic-carbon-rich sediment most commonly interbedded with black shale layers. The contrast in the organic carbon contents of these different lithologies is striking (Tables 2 and 4, Fig. 5). There are two possible interpretations for the green coloration: either it is the result of diagenetic reduction of iron in red muds deposited next to layers rich in organic matter, or it is from iron reduction during deposition of sediments in poorly oxygenated bottom waters. These 
Table 4. Summary of organic matter contents and paleoproductivities of Site 530 sediments and rocks.

\begin{tabular}{|c|c|c|c|c|c|c|c|c|c|c|}
\hline \multirow{2}{*}{$\begin{array}{l}\text { Lithologic } \\
\text { Unita }^{\mathrm{a}}\end{array}$} & \multirow{2}{*}{$\begin{array}{l}\text { Depth } \\
\text { (m) }\end{array}$} & \multirow{2}{*}{$\begin{array}{c}\text { Age } \\
\text { (m.y. ago) }\end{array}$} & \multirow[b]{2}{*}{ Description } & \multirow{2}{*}{$\begin{array}{l}\text { Sedimen- } \\
\text { tation } \\
\text { rate } \\
(\mathrm{m} / \mathrm{m} . \mathrm{y} .)\end{array}$} & \multicolumn{2}{|c|}{$\% \mathrm{C}_{\text {org }}$} & \multicolumn{2}{|c|}{ Atomic $\mathrm{C} / \mathrm{N}$} & \multirow{2}{*}{$\begin{array}{l}\text { No. of } \\
\text { samples }\end{array}$} & \multirow{2}{*}{$\begin{array}{l}\text { Paleopro- } \\
\text { ductivity } \\
\left(\mathrm{g} \mathrm{C} / \mathrm{m}^{2} / \mathrm{y} \text {.) }\right.\end{array}$} \\
\hline & & & & & Mean & Range & Mean & Range & & \\
\hline $1 \mathrm{a}$ & $0-58$ & $<1.7$ & $\begin{array}{l}\text { Diatom nannofossil marl and ooze and } \\
\text { debris-flow deposits }\end{array}$ & 65 & 3.04 & $0.79-4.81$ & 13.3 & $7.8-17.5$ & 25 & 150 \\
\hline $1 \mathrm{~b}$ & $58-110$ & $<1.7$ & Diatom ooze and debris-flow deposits & 65 & 3.68 & $2.10-6.40$ & 13.1 & $11.8-14.0$ & 7 & 182 \\
\hline 2 & $110-277$ & $1.7-10$ & $\begin{array}{l}\text { Nannofossil clay, marl, and ooze and } \\
\text { debris-flow deposits }\end{array}$ & 20 & 1.41 & $0.30-2.63$ & 11.1 & $5.6-24.1$ & 22 & 99 \\
\hline 3 & $277-467$ & $10-37$ & Red and green mud & 9 & 0.29 & $0.07-0.53$ & 5.5 & $2.1-11.3$ & 15 & 26 \\
\hline 4 & $467-600$ & $37-66$ & $\begin{array}{l}\text { Multicolored mudstone, marlstone, } \\
\text { chalk, and clastic limestone }\end{array}$ & 5 & 0.27 & $0.07-0.50$ & 8.4 & $5.3-12.4$ & 11 & 29 \\
\hline $5 a$ & $600-647$ & $66-69$ & $\begin{array}{l}\text { Dark green mudstone, marlstone, and } \\
\text { clastic limestone }\end{array}$ & 16 & 0.28 & $0.14-0.35$ & 9.3 & $6.0-14.4$ & 4 & 21 \\
\hline $5 \mathrm{~b}$ & $647-704$ & $69-70.5$ & $\begin{array}{l}\text { Dark green mudstone, marlstone, } \\
\text { clastic limestone, and siliciclastic } \\
\text { sandstone }\end{array}$ & 38 & 0.25 & $0.16-0.42$ & 11.9 & $5.4-15.1$ & 5 & 14 \\
\hline $5 \mathrm{c}$ & $704-790$ & $70.5-77.5$ & $\begin{array}{l}\text { Dark green mudstone, marlstone, and } \\
\text { calcareous siliciclastic sandstone }\end{array}$ & 11 & 0.20 & $0.11-0.26$ & 10.8 & $8.2-15.2$ & 3 & 17 \\
\hline 6 & $790-831$ & $77.5-80.5$ & Glauconitic sandstone & 20 & 0.26 & $0.15-0.37$ & 18.3 & $15.5-21.2$ & 2 & 18 \\
\hline 7 & $831-940$ & $80.5-84$ & $\begin{array}{l}\text { Variegated red and green claystone, } \\
\text { siltstone, and sandstone }\end{array}$ & 31 & 0.58 & $0.15-2.02$ & 16.0 & $9.0-21.3$ & 8 & 36 \\
\hline \multirow[t]{2}{*}{8} & $940-1103$ & $84-102.5$ & $\begin{array}{l}\text { Red and green claystone and marlstone } \\
\text { with interbedded black shale }\end{array}$ & 9 & 0.42 & $0.08-1.31$ & 12.4 & \multicolumn{3}{|c|}{$\begin{array}{l}\text { (red and green } \\
\text { claystones) }\end{array}$} \\
\hline & & & & & 5.37 & $0.57-16.5$ & 25.8 & $11.3-36.5$ & $\begin{array}{l}25 \\
\text { lack shales) }\end{array}$ & 480 \\
\hline
\end{tabular}

a Fully described in the Site 530 summary (this volume).

b Estimated from equation 7 of Müller and Suess (1979) (see text).

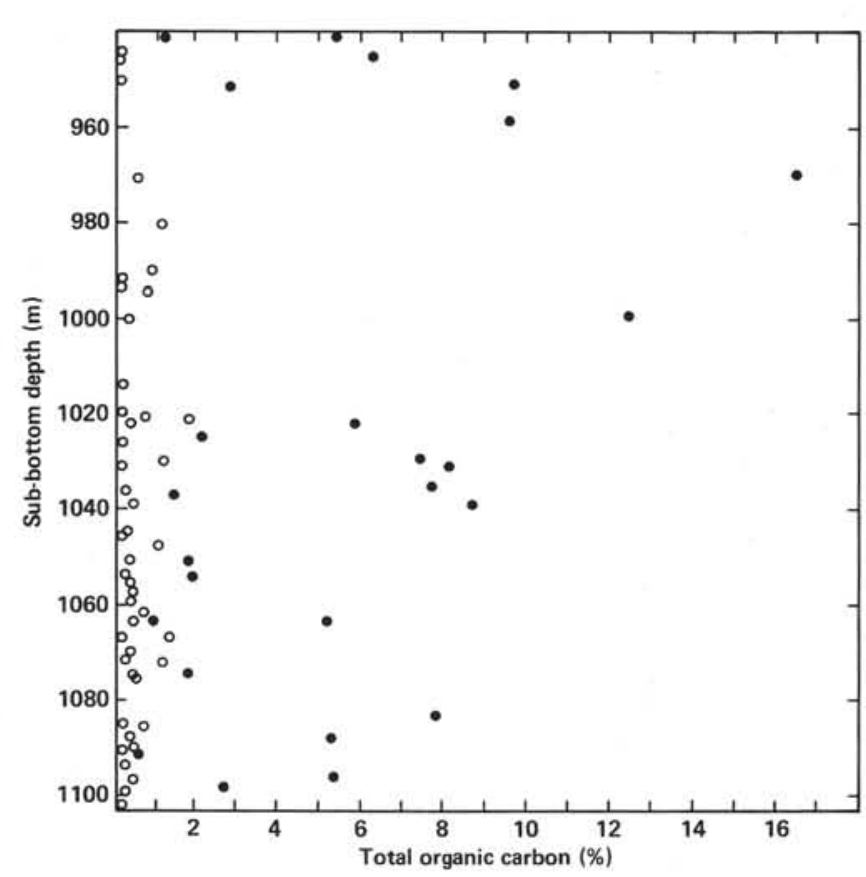

Figure 5. Comparison of concentrations of total organic carbon of black shales, green claystones, and red claystones from Unit 8, Hole 530A. (Black shale values are denoted as solid circles; red and green claystones as open circles.)

two interpretations are not mutually exclusive, and both processes may have operated. The frequent interbedding of red, green, and black layers, and the common bioturbation of much of the sediment but not in parts of the black shales, suggest that there was a delicate balance between oxidizing and reducing conditions in the sediments and waters of the Angola Basin during the Late Cretaceous.
It appears that the Angola Basin was sufficiently oxygenated during most of the Late Cretaceous to support an active benthic infauna. There were periods of shorter and longer duration when bottom-water conditions fluctuated between oxic and anoxic. Pelagic, hemipelagic, and turbiditic sedimentation processes continued during the anoxic periods, but deposits contained and preserved a higher concentration of organic matter, were relatively unbioturbated, and subsequently formed the black, organic-carbon-rich shales.

Lithologic Units 3 through 7 contain low amounts of organic matter (Table 4, Fig. 4), with mean organic carbon concentrations near the value of $0.2 \%$ typical of pelagic marine sediments (Degens and Mopper, 1976), and ranges similar to the range of 0.1 to $0.5 \%$ in DSDP Leg 58 sediments (Waples and Sloan, 1980). In Unit 7, the mean organic carbon value is elevated and the range is expanded by two samples of a Santonian black shale layer (Samples 530A-86-5, 31-32 cm and 33-34 cm); other samples in this unit average $0.22 \%$ organic carbon. These low concentrations are similar to the mean organic carbon value of $0.3 \%$ compiled by McIver (1975) from measurements done during DSDP Legs 1 through 31 and reflect a combination of low production and poor preservation of organic matter at Site 530 from the Santonian ( 84 m.y. ago) through the middle Miocene (10.5 m.y. ago).

Beginning in Unit 2, concentrations of organic carbon increase to a mean value of $3.68 \%$ in Unit $1 \mathrm{~b}$ before decreasing slightly to average $3.04 \%$ in Unit 1a (Table 4). Sediments of these Neogene units contain more biogenous components than do those deeper in the section, and their sedimentation rates increase to $65 \mathrm{~m} / 10^{6} \mathrm{y}$. in Pleistocene Units $1 \mathrm{a}$ and $1 \mathrm{~b}$. Paleoproductivity estimates increase from $99 \mathrm{~g} \mathrm{C} / \mathrm{m}^{2} / \mathrm{y}$., typical of coastal areas (Ryther, 1969) in Unit 2 to 150 to $182 \mathrm{~g} \mathrm{C} / \mathrm{m}^{2} / \mathrm{y}$. in 
Unit 1. These higher rates are similar to those for upwelling areas off northwest Africa (Müller and Suess, 1979). Higher sedimentation rates in the uppermost unit may have contributed to better preservation of organic matter, resulting in erroneously high estimates of paleoproductivity, but the increase in siliceous biogenic sediment components which accompanies the higher organic carbon values (Hay et al., 1982; Site 530 summary, this volume) indicates that the higher concentrations of organic carbon result from increased productivity since the mid to late Miocene.

Debris-flow deposits make up much of the thicknesses of Units 1 and 2 and represent downslope transport of biogenic sediments originally deposited on the Walvis Ridge south of Site 530 (Fig. 1). There are many similarities in organic carbon content, $\mathrm{C} / \mathrm{N}$ ratios, and lithologic composition between Neogene sediments from Site 530 (Table 2) and those from Site 532 (Table 3). Because the sediments at Site 530 appear to have had the same origin and history as sediments at Site 532, further discussion of them will be incorporated into the discussion of Site 532 .

\section{Site 532-Walvis Ridge}

The similarities in organic matter content of Neogene sediments from Sites 530 and 532 are shown in Figure 6. Values of organic carbon above the middle Miocene are substantially higher than the average DSDP value of $0.3 \%$ (McIver, 1975). Throughout these organic-carbon-rich layers, atomic $\mathrm{C} / \mathrm{N}$ ratios of organic matter remain ca. 15 despite variations in carbon concentration. Because changes in the degree of organic matter preservation would probably cause alterations of $\mathrm{C} / \mathrm{N}$ ratios (Müller, 1977; Waples and Sloan, 1980), the lack of such variability suggests that the fluctuations in amount of organic carbon result from changes in rate of input. These organic matter patterns may therefore record the history of biological productivity off southwest Africa, even though neither DSDP site is located under the present upwelling areas, which are generally closer to shore (Bishop et al., 1978).

Sedimentation rates and organic carbon contents of the Site 532 lithologic units can be used to estimate paleoproductivity rates since late Miocene times in waters overlying Walvis Ridge. Characteristics of the three lithologic sub-units recovered at Site $\mathbf{5 3 2}$ and their estimated paleoproductivities are given in Table 5. The highest paleoproductivity $\left(192 \mathrm{~g} \mathrm{C} / \mathrm{m}^{2} / \mathrm{y}\right.$.) occurs in Unit 1a (Pleistocene) and not in Unit $1 \mathrm{~b}$ (PleistocenePliocene), contrary to what would be expected from simple examination of organic carbon content, sediment lithology, and sedimentation rate. This discrepancy may arise because the expression developed by Müller and Suess (1979) adjusts for larger fractions of organic matter formed in surface waters becoming incorporated into the bottom under zones of greater productivity, but does not account for the later diagenetic losses described by Waples and Sloan (1980). The disagreement between actual sediment biogenic character and calculated rates of organic matter production suggests that the original level of organic carbon in Unit $1 \mathrm{~b}$ (and probably Unit
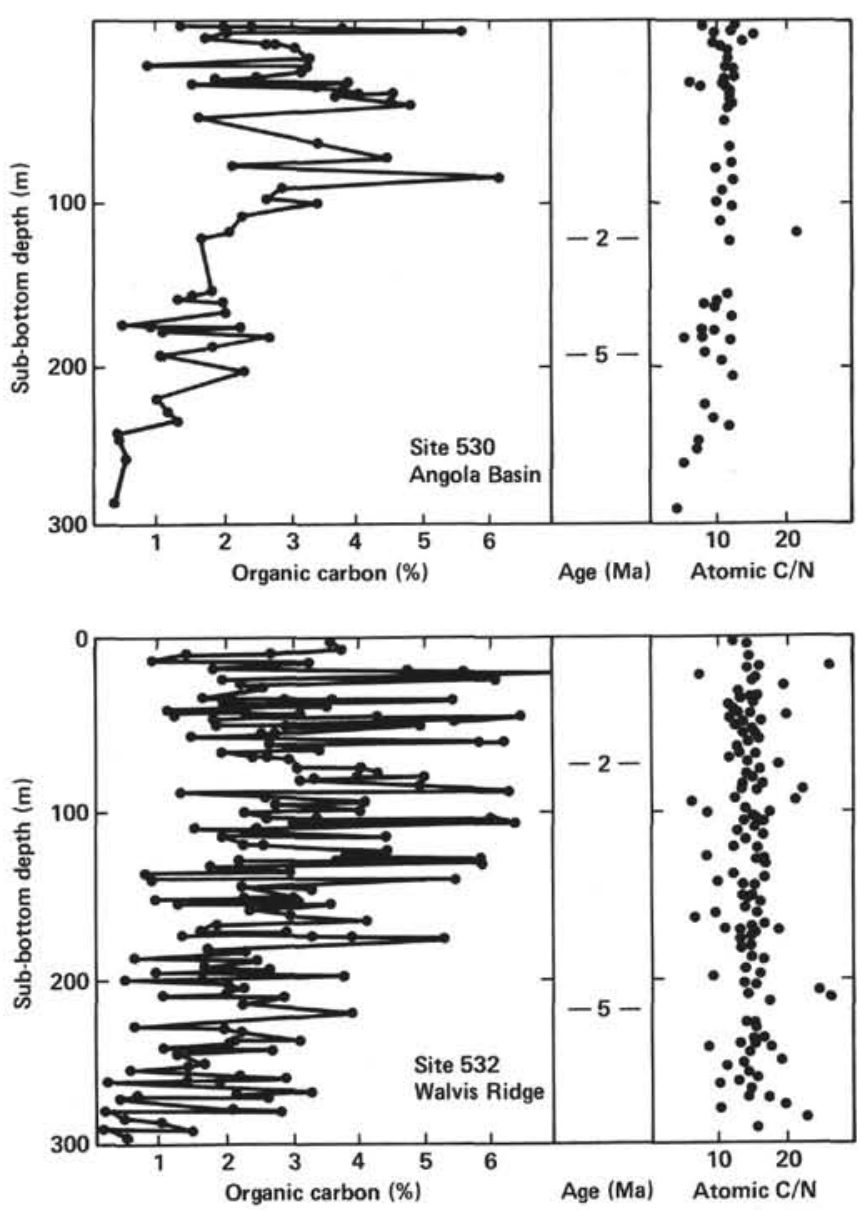

Figure 6. Organic carbon concentrations and atomic $\mathrm{C} / \mathrm{N}$ ratios of Neogene biogenic sediments from Sites 530 and 532. (Sediment ages are indicated in millions of years ago.)

1c) has been diminished by postdepositional degradation. Indeed, microbial activity to depths as great as ca. $200 \mathrm{~m}$ is indicated by copious amounts of biogenic gases in these sediments (Site 532 summary, this volume).

Siesser (1980) presents evidence from Site 362 adjacent to Site 532 on the Walvis Ridge that South Atlantic plankton changed from warm- to cold-water types in middle to late Miocene times. He interprets this as indicating the establishment of the modern Benguela Current system. As surface water temperatures decreased, surface productivity evidently increased in response to nutrient upwellings associated with the Benguela system. Information from Sites 530 and 532 supports the conclusions that the onset of the Benguela upwelling system occurred in the late Miocene (Diester-Haass and Schrader, 1979; Siesser, 1980). Because soft-sediment sampling at both sites was done by hydraulic piston corer, much of the sediment disturbance that accompanied the rotary drilling at Site 362 was avoided, and a more detailed sedimentary record of the history of this current system is available.

The strong similarity in patterns of biogenic sediment components between Sites 530 and 532 (Fig. 6) indicates a late Miocene onset and a late Pliocene maximum of upwelling off southwest Africa. At Site 530, turbidites 
Table 5. Summary of organic matter contents and paleoproductivities of Site 532 sediments.

\begin{tabular}{|c|c|c|c|c|c|c|c|c|c|c|}
\hline \multirow{2}{*}{$\begin{array}{l}\text { Lithologic } \\
\text { unit }^{\mathrm{a}}\end{array}$} & \multirow{2}{*}{$\begin{array}{l}\text { Depth } \\
(\mathrm{m})\end{array}$} & \multirow[b]{2}{*}{ Age } & \multirow[b]{2}{*}{ Description } & \multirow{2}{*}{$\begin{array}{l}\text { Sedimen- } \\
\text { tation } \\
\text { rate } \\
\text { (m/m.y.) }\end{array}$} & \multicolumn{2}{|c|}{$\% \mathrm{C}_{\text {org }}$} & \multicolumn{2}{|c|}{ Atomic $\mathrm{C} / \mathrm{N}$} & \multirow{2}{*}{$\begin{array}{c}\text { No. of } \\
\text { samples }\end{array}$} & \multirow{2}{*}{$\begin{array}{l}\text { Paleopro- } \\
\text { ductivity } \\
\left(\mathrm{g} \mathrm{C} / \mathrm{m}^{2} / \mathrm{y} .\right)\end{array}$} \\
\hline & & & & & Mean & Range & Mean & Range & & \\
\hline la & $0-50$ & Pleistocene & $\begin{array}{l}\text { Diatom nanno-foram } \\
\text { ooze and marl }\end{array}$ & 40 & 3.36 & $0.99-9.75$ & 14.5 & $4.8-26.3$ & 30 & 192 \\
\hline $\mathrm{lb}$ & $50-114$ & $\begin{array}{l}\text { Pleistocene- } \\
\text { Pliocene }\end{array}$ & $\begin{array}{l}\text { Nannofossil diatom } \\
\text { smarl and marl }\end{array}$ & 62 & 3.54 & $1.36-6.41$ & 14.2 & $4.7-21.7$ & 37 & 177 \\
\hline Ic & 114-291 & $\begin{array}{l}\text { Pliocene- } \\
\text { late Miocene }\end{array}$ & Nannofossil marl & 25 & 2.25 & $0.10-5.87$ & 14.7 & $5.9-30.1$ & 84 & 148 \\
\hline
\end{tabular}

a Fully described in the Site 532 summary (this volume).

b Estimated from equation 7 of Muller and Suess (1979) (see text).

and debris-flow deposits rich in organic matter and in diatoms began to accumulate in the late Miocene and became more extensive through the Pliocene. These deposits are poor in carbonate because of proximity to the calcite compensation depth, yet otherwise closely resemble the sediments found at Site $\mathbf{5 3 2}$ on the Walvis Ridge. The fact that they did not begin to accumulate in the Angola Basin until late Miocene times means that abundant amounts of biogenic sediments subject to slumping were not being formed before that time. Because of the steep northern slope of the Walvis Ridge (see Fig. 1), downslope transport to the Angola Basin and subsequent reburial must have been rapid; otherwise organic matter would not be so well preserved.

Sediments from Sites 530 and 532 contain considerable variability in organic carbon concentrations (Fig. 6 ), with periodicities of 30,000 to 50,000 years (Hay et al., 1982; Site 532 summary). Other cyclic patterns have been observed in marine sediments. Dean et al. (1981) report 44,000-year periodicities in Oligocene-Miocene biogenic sediments from the Sierra Leone Rise off northwest Africa. Rea (1982) infers 42,000-year cycles in the intensity of Pleistocene tradewinds over the eastern equatorial Pacific from variations in grain size of the eolian sedimentary component. This periodicity corresponds to the tilt cycle of the Earth, which affects the global distribution of incident solar radiation. Wind intensities shift in response to changes in latitudinal temperature gradient and may cause a similar periodicity in marine productivity (Rea, 1982). The cyclicity in biogenic sediment composition which is best preserved at Site 532 may reflect episodes of stronger winds and enhanced upwelling.

\section{SUMMARY}

A series of thin black shale layers containing up to $16.5 \%$ organic carbon are interbedded in Albian to Coniacian organic-carbon-lean red and green claystones at Site 530 in the Angola Basin. The conditions which led to formation of the black shales required a combination of high biological productivity and enhanced preservation of organic matter. Presence of closely associated red claystones and evidence of burrowing argue against long periods of bottom-water anoxia in the Late Cretaceous Angola Basin, but do not preclude short periods of mildly anoxic conditions nor an expanded and intensified midwater oxygen minimum zone. The same set of conditions need not have prevailed through- out the sediments deposited at Site 530 nor throughout the Cretaceous Atlantic Ocean.

Neogene biogenic ooze recovered by hydraulic piston coring at Sites 530 and 532 contains high concentrations of high organic carbon which began to accumulate during the late Miocene and records the onset of upwelling off southwest Africa. Productivity increased to a maximum in the late Pliocene and has declined somewhat since that time. Cycles of lower and higher productivity have periodicities of 30,000 to 50,000 years and may reflect variations in global wind intensities related to the tilt cycle of the Earth.

\section{ACKNOWLEDGMENTS}

We thank W. E. Dean, D. K. Rea, and C. P. Summerhayes for their thoughtful and helpful reviews of this paper. Analysis of the large number of samples was possible only with the help of W. Meyer and M. Nohara onboard D/V Glomar Challenger and of R. M. Glover, P. A. Pulver, M. S. Williams, M. J. Leenheer, and O. E. Kawka at The University of Michigan.

\section{REFERENCES}

Arthur, M. A., and Natland, J. H., 1979. Carbonaceous sediments in the North and South Atlantic: The role of salinity in stable stratification of Early Cretaceous basins. In Talwani, M., Hay, W., and Ryan, W. B. F., (Eds.), Deep Drilling Results in the Atlantic Ocean: Continental Margins and Paleoenvironment: Washington (Am. Geophys. Union), pp. 375-401.

Bishop, J. K. B., Ketten, D. R., and Edmond, J. M., 1978. The chemistry, biology, and vertical flux of particulate matter from the upper $400 \mathrm{~m}$ of the Cape Basin in the southeast Atlantic Ocean. Deep-Sea Res., 25:1121-1161.

Dean, W. E., Gardner, J. V., and Čepek, P., 1981. Tertiary carbonate-dissolution cycles on the Sierra Leone Rise, eastern equatorial Atlantic Ocean. Mar. Geol., 39:81-101.

Degens, E. T., and Mopper, K., 1976. Factors controlling the distribution and early diagenesis of organic material in marine sediments. In Riley, J. P., and Chester, R. (Eds.), Chemical Oceanography (Vol. 6): New York (Academic Press), 59-113.

Diester-Haass, L., and Schrader, H.-J., 1979. Neogene coastal upwelling history off northwest and southwest Africa. Mar. Geol., 29: 39-53.

Erdman, J. G., and Schorno, K. S., 1978. Geochemistry of carbon: Deep Sea Drilling Project Leg 40. In Bolli, H. M., Ryan, W. B. F., et al., Init. Repts. DSDP, 40: Washington (U.S. Govt. Printing Office), 651-658.

Firstbrook, P. L., Funnell, B. M., Hurley, A. M., and Smith, A. G., 1979. Paleoceanic Reconstructions: Washington (National Science Foundation).

Forster, R., 1978. Evidence for an open seaway between northern and southern proto-Atlantic in Albian times. Nature, 272:158-159.

Foresman, J. B., 1978. Organic geochemistry, DSDP Leg 40, continental rise of Southwest Africa. In Bolli, H. M., Ryan, W. B. F., et al., Init. Repts. DSDP, 40: Washington (U.S. Govt. Printing Office), 557-567. 
Goodell, H. G., 1972. Carbon/nitrogen ratio. In Fairbridge, R. W. (Ed.), Encyclopedia of Geochemistry and Environmental Science: New York (Van Nostrand Reinhold), pp. 136-142.

Hay, W. W., Sibuet, J.-C., and Leg 75 Shipboard Scientific Party, 1982. Sedimentation and accumulation of organic carbon in the Angola Basin and on Walvis Ridge: Preliminary results of Deep Sea Drilling Project Leg 75. Geol. Soc. Am. Bull., 93:1038-1058.

Heezen, B. C., and Hollister, C. D., 1971. The Face of the Deep: New York (Oxford University Press).

Leventhal, J. S., Crock, J. G., Mountjoy, W., Thomas, J. A., Shale, V. E., Briggs, P. H., Wahlberg, J. S., and Malcom, M. J., 1978. Preliminary analytical results for a new US Geological Survey Devonian shale standard, SDO-1. USGS Open File Report, 78-447.

McIver, R., 1975. Hydrocarbon occurrences from JOIDES Deep Sea Drilling Project. Proc. Ninth World Pet. Congr., Tokyo: London (Applied Science Publ.), 2:269-280.

Menzel, D. W., 1974. Primary productivity, dissolved and particulate organic matter, and the sites of oxidation of organic matter. In Goldberg, E. D. (Ed.), The Sea (Vol. 5): New York (Elsevier), 659-678.

Müller, G., and Gastner, M., 1971. The "karbonate bomb," a simple device for determination of the carbonate content in sediments, soils, and other materials. N. Jahrb. Mineral. Mh., 10:466-469.

Müller, P. J., 1977. C/N ratios in Pacific deep-sea sediments: Effect of inorganic ammonium and organic nitrogen compounds sorbed by clays. Geochim. Cosmochim. Acta, 41:765-776.
Müller, P. J., and Suess, E., 1979. Productivity, sedimentation rate, and sedimentary organic matter in the oceans-I. Organic carbon preservation. Deep-Sea Res., 26A:1347-1362.

Rea, D. K., 1982. Fluctuation in eolian sedimentation during the past five glacial-interglacial cycles: A preliminary examination of data from Deep Sea Drilling Project Hole 503B, eastern equatorial Pacific. In Prell, W. L., Gardner, J. V., et al., Init. Repts. DSDP, 68: Washington (U.S. Govt. Printing Office).

Ryther, J. H., 1969. Photosynthesis and fish production in the sea. Science, 166:72-76.

Siesser, W. G., 1980. Late Miocene origin of the Benguela upwelling system off northern Namibia. Science, 208:283-285.

Tissot, B., Deroo, G., and Herbin, J. P., 1979. Organic matter in Cretaceous sediments of the North Atlantic: Contributions to sedimentology and paleogeography. In Talwani, M., Hay, W., and Ryan, W. B. F. (Eds.), Deep Drilling Results in the Atlantic Ocean: Continental Margins and Paleoenvironment: Washington (Am. Geophys. Union), pp. 362-374.

Waples, D. W., and Sloan, J. R., 1980. Carbon and nitrogen diagenesis in deep sea sediments. Geochim. Cosmochim. Acta, 44: 1463-1470.

Date of Initial Receipt: May 19, 1982 